\title{
Do peaked solitary water waves indeed exist?
}

\author{
Shijun Liao ${ }^{1,2,3}$ \\ ${ }^{1}$ State Key Laboratory of Ocean Engineering, Shanghai 200240, China \\ 2 School of Naval Architecture, Ocean and Civil Engineering \\ Shanghai Jiaotong University, Shanghai 200240, China \\ ${ }^{3}$ Dept. of Mathematics, Shanghai Jiaotong University, Shanghai 200240, China
}

(Email address: sjliao@sjtu.edu.cn)

\begin{abstract}
Many models of shallow water waves, such as the famous Camassa-Holm equation, admit peaked solitary waves. However, it is an open question whether or not the widely accepted peaked solitary waves can be derived from the fully nonlinear wave equations. In this paper, a unified wave model (UWM) based on the symmetry and the fully nonlinear wave equations is put forward for progressive waves with permanent form in finite water depth. Different from traditional wave models, the flows described by the UWM are not necessarily irrotational at crest, so that it is more general. The unified wave model admits not only the traditional progressive waves with smooth crest, but also a new kind of solitary waves with peaked crest that include the famous peaked solitary waves given by the Camassa-Holm equation. Besides, it is proved that Kelvin's theorem still holds everywhere for the newly found peaked solitary waves. Thus, the UWM unifies, for the first time, both of the traditional smooth waves and the peaked solitary waves. In other words, the peaked solitary waves are consistent with the traditional smooth ones. So, in the frame of inviscid fluid, the peaked solitary waves are as acceptable and reasonable as the traditional smooth ones. It is found that the peaked solitary waves have some unusual and unique characteristics. First of all, they have a peaked crest with a discontinuous vertical velocity at crest. Especially, unlike the traditional smooth waves that are dispersive with wave height, the phase speed of the peaked solitary waves has nothing to do with wave height, but depends (for a fixed wave height) on its decay length, i.e. the actual wavelength: in fact, the peaked solitary waves are dispersive with the actual wavelength when wave height is fixed. In addition, unlike traditional smooth waves whose kinetic energy decays exponentially from free surface to bottom, the kinetic energy of the peaked solitary waves either increases or almost keeps the same. All of these unusual properties show the novelty of the peaked solitary waves, although it is still an open question whether or not they are reasonable in physics if the viscosity of fluid and surface tension are considered.
\end{abstract}

Key words unified wave model (UWM), progressive wave, solitary peaked wave, homotopy analysis method (HAM) 


\section{Introduction}

Since the solitary surface wave was discovered by John Scott Russell [1] in 1834, various types of solitary waves have been found. The mainstream models of shallow water waves, such as the Boussinesq equation [2], the KdV equation [3], the BBM equation [4] and so on, admits dispersive smooth periodic and solitary waves with permanent form: the wave elevation is infinitely differentiable everywhere. Especially, the phase speed of these smooth waves is closely related to wave height: a smooth progressive wave with higher amplitude always propagates faster. Today, such kind of amplitude-dispersive periodic and solitary waves with smooth crest are the mainstream of water waves.

However, the discontinuity of water wave elevation appears accidentally in theory. It is well-known that the famous limiting gravity wave has a corner crest with 120 degree, as pointed out by Stokes [5] in 1894. It is a pity that the importance of such kind of discontinuity seems to be neglected, mainly because Stokes' limiting gravity wave [5] has been never observed in practice. In 1993, almost one hundred years later, Camassa \& Holm [6] proposed a model for shallow water waves with small amplitude, called today the Camassa-Holm $(\mathrm{CH})$ equation

$$
u_{t}+2 \omega u_{X}-u_{X X t}+3 u u_{X}=2 u_{X} u_{X X}+u u_{X X X}
$$

where $u(X, t)$ denotes the wave elevation, $X$ and $t$ are the spatial and temporal variables, $\omega$ is a constant related to the critical phase speed of shallow water waves, respectively. As pointed out by Camassa \& Holm [6], the $\mathrm{CH}$ equation (1) has the solitary wave when $0 \leq \omega<1 / 2$. Especially, in the limit $\omega \rightarrow 0$, the $\mathrm{CH}$ equation (1) admits the peaked solitary wave in the closed-form

$$
u(X, t)=c \exp (-|X-c t|),
$$

whose first derivative is discontinuous at the crest $X=c t$, where $c$ denotes the phase speed. Unlike the $\mathrm{KdV}$ equation and Boussinesq equation, the $\mathrm{CH}$ equation (1) can model phenomena of soliton interaction and wave breaking, as mentioned by Constantin [7]. Mathematically, the $\mathrm{CH}$ equation (11) is integrable and bi-Hamiltonian, thus possesses an infinite number of conservation laws in involution, as pointed out by Camassa \& Holm [6]. In addition, it is associated with the geodesic flow on the infinite dimensional Hilbert manifold of diffeomorphisms of line, as mentioned by Constantin [7]. Thus, the $\mathrm{CH}$ equation (1) has many intriguing physical and mathematical properties. A few researchers even believed that the $\mathrm{CH}$ equation (1) "has the potential to become the new master equation for shallow water wave theory" [8].

Note that the discontinuity also widely appears in practical flows, such as dam break in hydrodynamics and shock wave in aerodynamics. In fact, such kind of discontinuous problems belong to the so-called Riemann problem [9,10], a classic research field. So, the discontinuity of wave elevation seems to be reasonable not only in mathematics but also in physics. 
Thousands of articles for peaked solitary waves have been published. Currently, the closed-form solutions of peaked solitary waves of the Boussinesq equation, the KdV equation, the BBM equation, the modified KdV equation and KP equation are derived by Liao [11,12] using the symmetry and the corresponding wave models mentioned above. Besides, Kraenkel and Zenchuk [13] gave the explicit cusped solitary waves of the $\mathrm{CH}$ equation (1) when $\omega \neq 0$, called cuspon. The so-called cuspon is a kind of solitary wave with the 1st derivative going to infinity at crest. Note that the peakon has a finite 1st derivative, but the cuspon has an infinite 1st derivative at crest. Thus, peakons and cuspons are completely different two kinds of discontinuous solutions of the $\mathrm{CH}$ equation (11). Therefore, nearly all mainstream models of the shallow water waves admit the peaked solitary waves, as pointed out by Liao [11,12].

However, there are many open questions about peaked solitary waves. Where does this kind of discontinuity come from? Are these peaked solitary waves as acceptable and reasonable as the traditional smooth ones? Are there any other peaked waves in finite water depth? It should be emphasized that all of the above-mentioned mainstream models of shallow water waves are approximations of the fully nonlinear wave equations under assumptions of the existence of some small physical parameters (such as small wave amplitude, shallow water and so on). Can we gain the peaked solitary waves from the fully nonlinear water wave equations? To the best of the author's knowledge, this is still an open question up to now. This situation is rather strange. Logically, if the peaked solitary waves given by the simplified wave models are acceptable and reasonable, the exact fully nonlinear wave equations should also admit it. Otherwise, we must check carefully the acceptableness and reasonableness of the peaked/cusped solitary waves reported/studied in thousands of published articles.

In this article, a positive answer to this open question is given. Proposing a unified wave model (UWM) based on the symmetry and the fully nonlinear wave equations, we gain a new type of solitary surface waves in finite water depth, which have a peaked crest and possess many unusual characteristics quite different from the traditional smooth ones. The UWM admits two different kinds of waves: one is infinitely differentiable everywhere, with a phase speed closely related to the wave height, the other has a peaked crest and especially the discontinuous vertical velocity at crest, with a phase speed having nothing to do with wave height! The former are exactly the same as the traditional periodic and solitary waves with smooth crest found in textbooks. However, the latter has many completely new properties and has never been reported, to the best of our knowledge. In theory, the UWM unifies both of the traditional smooth waves and the peaked solitary waves, for the first time. Therefore, in essence, the peaked solitary waves are consistent with the traditional smooth ones, and thus are as acceptable and reasonable as them.

This article is outlined as follows. In $\S$ 2, a unified wave model (UWM) based on the symmetry and the fully nonlinear wave equations for progressive gravity waves with permanent form in finite water depth is described, which admits not only the traditional periodic and solitary progressive waves with smooth crest (see $\S 3$ ), but also a new type of peaked solitary waves (see $\S 4$ ). In $\S 4.1$, a new type of peaked solitary surface waves are first obtained by means of the linearized UWM. In $\S 4.2$, 
such kind of peaked solitary surface waves are confirmed by the exact UWM. This kind of peaked solitary surface waves have some unusual characteristics quite different from traditional smooth ones, as described in $\S 4.3$. Besides, it is proved in $\S 4.4$ that the Kelvin's theorem still holds everywhere for these peaked solitary waves. The concluding remarks and discussions about the newly found peaked solitary waves are given in $\S$ 5. The corresponding nonlinear partial differential equations are solved in $\S 4.2$ by means of an analytic approximation method for highly nonlinear problems, namely the homotopy analysis method (HAM) [14-20], which has nothing to do with small/large physical parameters at all and besides can guarantee the convergence of solution series.

\section{A unified wave model based on symmetry}

First of all, we describe a unified wave model (UWM) for progressive waves with permanent form in water of finite depth. Note that, mathematically, we must be extremely careful so that the discontinuous solutions are not lost.

Consider a progressive surface gravity wave propagating on a horizontal bottom with a constant phase speed $c$ and a permanent form in a finite water depth $D$. For simplicity, let us consider the problem in the frame moving with the phase speed $c$. Let $x, z$ denote the horizontal and vertical dimensionless co-ordinates (using $D$ as the characteristic length), with $x=0$ corresponding to the wave crest, $z=-1$ to the bottom, and the $z$ axis upward, respectively. Assume that the wave elevation has a symmetry about the crest at $x=0$. Due to this symmetry, we need consider the interval $x \geq 0$ only. Assume that the fluid in the domain $x>0$ is inviscid and incompressible, the flow is irrotational, and surface tension is neglected. It should be emphasized here that the flow at $x=0$ is not necessarily irrotational. In other words, there might exist the vorticity at $x=0$. Thus, this model based on the symmetry is more general than others, as shown below.

Let $g$ denote the acceleration due to gravity, $\phi$ the velocity potential (in the interval $0<x<+\infty)$, $\zeta$ the free surface, respectively. All of these variables are dimensionless by means of $D$ and $\sqrt{g D}$ as the characteristic scales of length and velocity. Due to the symmetry $\zeta(-x)=\zeta(x)$, we only need consider the flow in the interval $x \in(0,+\infty)$. Since the fluid in the domain $x>0$ is inviscid and incompressible, besides the flow is irrotational and the surface tension is neglected, it is easy to have the governing equation

$$
\nabla^{2} \phi(x, z)=0, \quad z \leq \zeta(x), 0<x<+\infty,
$$

subject to the boundary conditions on the unknown free surface $z=\zeta(x)$ :

$$
\begin{gathered}
\alpha^{2} \frac{\partial^{2} \phi}{\partial x^{2}}+\frac{\partial \phi}{\partial z}-\alpha \frac{\partial}{\partial x}(\nabla \phi \cdot \nabla \phi)+\nabla \phi \cdot \nabla\left(\frac{1}{2} \nabla \phi \cdot \nabla \phi\right)=0, \quad 0<x<+\infty, \\
\zeta-\alpha \frac{\partial \phi}{\partial x}+\frac{1}{2} \nabla \phi \cdot \nabla \phi=0, \quad 0<x<+\infty
\end{gathered}
$$


and the bottom condition

$$
\frac{\partial \phi}{\partial z}=0, \quad z=-1, \quad 0<x<+\infty,
$$

where $\nabla^{2}$ is a Laplace operator, and

$$
\alpha=\frac{c}{\sqrt{g D}}
$$

is the dimensionless wave-speed, respectively. Note that all of the above-mentioned governing equation and boundary conditions are defined in the domain $0<x<+\infty$. In fact, they are just the fully nonlinear wave equations, except the restricted interval $0<x<+\infty$. Besides, we have either the periodic condition

$$
\phi(x, z)=\phi(x+\lambda, z), \quad-1 \leq z \leq \zeta(x)
$$

for the periodic progressive waves, where $\lambda=2 \pi / k$ is the wavelength, or the decay condition

$$
\phi( \pm \infty, z)=0, \quad-1 \leq z \leq \zeta(x)
$$

for the solitary waves. On the vertical boundary $x=0$, we have the additional condition

$$
u(0, z)=\lim _{x \rightarrow 0} \frac{\partial \phi}{\partial x}=U(z), \quad z \leq \zeta(x),
$$

where $U(z)$ is such an unknown horizontal velocity at $x=0$ that the corresponding velocity potential $\phi(x, z)$ and the progressive wave elevation $\zeta(x)$ with permanent form exist. Furthermore, let $H_{w}$ denote the dimensionless wave-elevation at $x=0$, corresponding to the wave crest. Then, for given $H_{w}$, one has an addition condition

$$
\lim _{x \rightarrow 0} \zeta(x)=H_{w} .
$$

In addition, the wave elevation must be bounded, i.e.

$$
|\zeta(x)|<C, \quad 0<x<+\infty,
$$

for a large enough constant $C$.

Let $u(x, y)$ and $v(x, y)$ denote the horizontal and vertical velocity of fluid, respectively. Due to the symmetry, in the domain $x \in(-\infty,+\infty)$, we have the symmetry

$$
\zeta(x)=\zeta(-x), \quad u(x, z)=u(-x, z), \quad v(x, z)=-v(-x, z),
$$

which gives at the crest $(x=0)$ that

$$
v(0, z)=-v(0, z) \text {, i.e. } v(0, z)=0 .
$$

Since the flow is irrotational in the interval $x \in(0,+\infty)$, the corresponding velocities $u(x, z)$ and $v(x, z)$ are given by

$$
u(x, z)=\frac{\partial \phi}{\partial x}, \quad v(x, z)=\frac{\partial \phi}{\partial z}, \quad 0<x<+\infty .
$$


Note that, at crest $(x=0)$, we have the boundary condition $v=0$ due to the symmetry, and the boundary condition $u=U(z)$ defined by the limit (10) as $x \rightarrow 0$. It should be emphasized that, in order not to lose the solutions that are discontinuous at crest $(x=0)$, the boundary condition (10) is defined by a limit. In this way, the problem is described more generally than the traditional theories.

Note that, according to the symmetry (13) about crest (at $x=0)$, the wave elevation $\zeta(x)$ and the horizontal velocity $u$ are continuous at the vertical boundary $x=0$. In fact, due to the symmetry (13), the boundary condition (10) is equivalent to the continuous condition of the horizontal velocity $u$ at $x=0$. It is a common knowledge that the Laplace equation (3) needs only one boundary condition at each boundary. Therefore, at $x=0$, the boundary condition (10) is sufficient for the Laplace equation (3) so that any other conditions for the smoothness of the horizontal velocity $u($ as $x \rightarrow 0)$ are unnecessary: the higher-order derivatives of the horizontal velocity

$$
\lim _{x \rightarrow 0} \frac{\partial^{2} \phi}{\partial x^{2}}, \lim _{x \rightarrow 0} \frac{\partial^{3} \phi}{\partial x^{3}}, \lim _{x \rightarrow 0} \frac{\partial^{4} \phi}{\partial x^{4}}, \cdots,
$$

are unnecessary to be continuous, since the boundary condition (10) is mathematically enough for the Laplace equation (3). Thus, any other boundary conditions (such as that $\phi$ and $\zeta$ should be infinitely differentiable at $x=0$ ) may lead to the loss of the discontinuous solutions and thus must be avoided. In other words, in the frame of the UWM, both of $\phi$ and $\zeta$ are unnecessary to be infinitely differentiable at crest $(x=0)$. This is mainly because the velocity potential $\phi$ satisfies Laplace equation only in the domain $x>0$ and $x<0$ so that $\phi$ is smooth only in $x>0$ or $x<0$, but not necessarily analytic at $x=0$.

The two nonlinear boundary conditions (44) and (5) must be satisfied on the unknown free surface $z=\zeta(x)$. This leads to the mathematical difficulty to solve the nonlinear partial differential equations (PDEs). In case of small wave-amplitude, the linear boundary condition

$$
\alpha^{2} \frac{\partial^{2} \phi}{\partial x^{2}}+\frac{\partial \phi}{\partial z}=0, \quad \text { on } z=0,0<x<+\infty,
$$

is a good approximation of (44), and

$$
\zeta(x)=\left.\alpha \frac{\partial \phi}{\partial x}\right|_{z=0}, \quad 0<x<+\infty,
$$

is a good approximation of (5), respectively. The above two linearized free-surface boundary conditions, combined with the Laplace equation (3), the bottom condition (6) and either the periodic condition (8) or the decay condition (9), provide us the so-called linear UWM.

\section{Smooth gravity waves given by the UWM}

Based on the traditional linear or fully nonlinear wave equations, thousands of articles have been published for the smooth periodic and solitary progressive waves. Mostly, 
these traditional smooth progressive waves are based on the base functions

$$
\cosh [n k(z+1)] \sin (n k x), \quad n \geq 1
$$

for the velocity potential $\phi$, which automatically satisfy the Laplace equation (3), the bottom condition (6) and the periodic condition (8), where $k$ denotes the wave number and $n \geq 1$ is an integer. For smooth periodic progressive waves with small wave-amplitude, substituting the velocity potential

$$
\phi(x, z)=\alpha A_{0} \cosh [k(z+1)] \sin (k x)
$$

into the linear boundary condition (15), one has the dimensionless phase speed

$$
\alpha=\sqrt{\frac{\tanh (k)}{k}} \leq 1
$$

say, the phase speed of a smooth periodic progressive wave in a finite water depth $D$ is always less then $\sqrt{g D}$. Besides, substituting (18) into (16) gives the wave elevation with small amplitude

$$
\zeta=\frac{H_{w}}{2} \cos (k x)
$$

where $H_{w}=2 A_{0} \sinh (k)$. The corresponding horizontal velocity reads

$$
u(x, z)=\frac{H_{w}}{2 \alpha \cosh (k)} \cosh [k(z+1)] \cos (k x),
$$

which gives

$$
\frac{u}{U_{0}}=\frac{\cosh [k(z+1)] \cos (k x)}{\cosh (k)}, \quad 0<x<+\infty,
$$

where $U_{0}=H_{w} /(2 \alpha)$. At $x=0$, we have the corresponding horizontal velocity

$$
U(z)=\lim _{x \rightarrow 0} u(x, z)=\frac{U_{0}}{\cosh (k)} \cosh [k(z+1)] .
$$

Note that the above velocity potential (18) and wave elevation (20) automatically satisfy the symmetry (13). Besides, at $x=0$, the horizontal velocity $u=U(z)$ is gained by the limit (10), and the boundary condition $v=0$ is automatically satisfied. In other words, first solving the linearized PDEs (3) - (12) in the interval $0<x<+\infty$ and then expanding the results to the interval $-\infty<x<0$ by means of the symmetry (13), we can gain exactly the same results by the linear UWM as the traditional ones, i.e. the Airy wave. In this case, the velocity potential $\phi$ and the wave elevation are smooth everywhere. All of these indicate that the linear UWM is consistent with the traditional theories for linear smooth periodic progressive waves.

Note that, for given $x$, the horizontal velocity $u$ and the kinetic energy of the smooth periodic progressive Airy's waves decrease exponentially from free surface $(z=0)$ to the bottom $(z=-1)$. Especially, based on the base functions (17), 
the elevation of the Airy's wave and the corresponding velocities are infinitely differentiable everywhere, although the UWM does not contain any smoothness conditions at all.

For the traditional periodic progressive surface waves with large amplitude, the fully nonlinear wave equations must be considered. As pointed out by Cokelet [21], the phase speed $c$ of the smooth periodic progressive waves depends not only on the water depth $D$ and the wave number $k$ but also on the wave height $H_{w}$ : the larger the wave amplitude, the faster the periodic wave propagates. In other words, the smooth periodic progressive waves are dispersive with wave height. Like the linear Airy waves, the nonlinear periodic progressive surface waves have a smooth crest with the exponentially decaying velocity $u(x, z)$ and kinetic energy from free surface to bottom. Besides, they are also infinitely differentiable everywhere, although the boundary conditions of such kind of smoothness do not exist at all for all traditional wave theories.

It is very interesting that, using the UWM, i.e. first solving the fully nonlinear wave equations (3) $-(12)$ in the interval $0<x<+\infty$ and then expanding the results to the interval $-\infty<x<0$ by means of the symmetry (13), we can gain exactly the same results as the traditional nonlinear periodic progressive waves. Besides, the corresponding boundary condition $v=0$ is satisfied automatically at crest $(x=0)$. To shorten the length of this article, we neglect the detailed mathematical derivation for them. Here, we only emphasize that the UWM indeed admits the traditional nonlinear periodic progressive waves with smooth crest.

It should be emphasized that, in the frame of the traditional linear wave theories, solitary waves have never be reported, to the best of the author's knowledge. For details, please refer to Mei et al. [22]. Solitary waves for nonlinear and amplitudedispersive long waves had been found by Boussinesq [2] and Rayleigh [23]. For amplitude-dispersive long waves of permanent form, the so-called KdV equation [3] gives the periodic cnoidal wave for a finite wavelength $\lambda$, which tends to the solitary wave

$$
\zeta(x)=H_{w} \operatorname{sech}^{2}\left[\frac{\sqrt{3 H_{w}}}{4}(x-c t)\right]
$$

with the phase speed

$$
c=\sqrt{1+H_{w}}
$$

as $\lambda \rightarrow+\infty$. Both of the above-mentioned solitary wave and the nonlinear periodic progressive waves are special cases of the so-called cnoidal waves. By means of perturbation methods and using the fully nonlinear wave equations, Fenton [24,25] gave a high-order cnoidal wave theory and a ninth-order solution for the solitary wave in the form

$$
\zeta(x)=\sum_{i=1}^{+\infty} \sum_{j=1}^{i} a_{i, j} \epsilon^{i}\left[\operatorname{sech}^{2}(\beta x)\right]^{j},
$$

where $a_{i, j}, \epsilon, \beta$ are constants. It should be emphasized that all of these traditional cnoidal and solitary waves have a smooth crest: $\zeta(x)$ is infinitely differentiable everywhere. Besides, the horizontal velocity $u(x, z)$ and the kinetic energy always decay 
exponentially from free surface to bottom. Furthermore, the phase speed is dependent upon wave height, say, the traditional cnoidal and solitary waves are dispersive with wave height. Finally, to the best of author's knowledge, all traditional solitary surface waves have a crest higher than the still water: solitary waves in the form of depression have been reported only for interfacial waves, but never for the surface waves.

It is very interesting that, using the UWM, i.e. first solving the PDEs (3) - (12) (or the $\mathrm{KdV}$ equation) in the domain $0<x<+\infty$ in a traditional way and then expanding these results to the domain $-\infty<x<0$ by means of the symmetry (13), we can gain exactly the same results as the traditional cnoidal and solitary waves with smooth crest! Especially, the boundary condition $v=0$ at crest $(x=0)$ and the required smoothness in the whole domain are automatically satisfied. This is mainly because the corresponding base functions such as (17) are smooth everywhere, and besides automatically satisfy the symmetry condition (13) and the boundary condition $v=0$ at crest. To shorten the length of this article, the detailed mathematical derivations are neglected here. All of these indicate that the UWM based on the symmetry (13) and the fully nonlinear wave equations (3) - (12) is indeed consistent with all traditional theories for the smooth progressive waves with permanent form.

Indeed, the traditional periodic and solitary progressive waves are smooth everywhere. However, this kind of smoothness is not absolutely necessary, since all of these traditional smooth waves can be obtained in the frame of the traditional wave theories and the UWM without any smoothness conditions at all! In essence, such kind of perfect smoothness of the wave elevation and velocities come automatically from the base functions (17), which are infinitely differentiable everywhere.

In summary, the unified wave model (UWM) admits all traditional smooth periodic and solitary progressive waves with permanent form! Thus, the UWM based on the symmetry and the fully nonlinear wave equations defined in $0<x<+\infty$ is consistent with the traditional wave theories defined in the whole domain $-\infty<x<+\infty$. This shows the generality of the UWM.

\section{Peaked solitary waves given by the UWM}

In this section, we illustrate that the unified wave model (UWM) based on symmetry (13) and the fully nonlinear wave equations also admits the peaked solitary waves, which include the famous peaked solitary wave (2) of the $\mathrm{CH}$ equation (1) but have many unusual characteristics quite different from the traditional smooth ones. Thus, the UWM unifies the traditional smooth waves and the peaked solitary ones, for the first time, to the best of the author's knowledge.

Mathematically, like the base functions (17) that are widely used for the smooth periodic and solitary progressive waves, the following base functions

$$
\cos [n k(z+1)] \exp (-n k x), \quad n \geq 1, k>0,0 \leq x<+\infty
$$

also automatically satisfy the Laplace equation (3) in the domain $0<x<+\infty$, 
the bottom condition (6) and the bounded condition (12), too. However, different from the smooth base functions (17), the above base function decays exponentially in the $x$ direction and satisfies the decay boundary condition (9) at infinity. Thus, it is more convenient to strictly express solitary waves that have truly only one crest. Note that, unlike the base function (17), its derivatives with respect to $x$ are not differentiable at crest $(x=0)$. Even so, the base function (26) with discontinuity of the 1st-derivative at crest was widely used as the so-called evanescent (or nonpropagating) mode [26] in the problems of linear water wave diffraction-refraction by discontinuous bed undulations [27 30], or linear waves propagating over a bed consisting of substantial variations in water depth [31-33], and so on. The solutions of these problems contain not only the propagating waves expressed by the base function (17), but also the non-propagating (or evanescent) waves expressed by the base function (26), which represent localized effects and depend on the local bottom geometry 33 . However, to the best of the author's knowledge, the evanescent base functions (26) have never been applied to express progressive solitary waves with permanent form propagating in a constant, finite water depth.

Can we find any peaked solitary waves in the frame of the UWM based on the symmetry and the fully nonlinear wave equations (31) - (12) by means of the evanescent base function (26)? The answer is positive: the UWM indeed admits a kind of solitary surface waves with peaked crest, which not only include the famous solitary wave (2) of the $\mathrm{CH}$ equation (1) but also have many unusual characteristics quite different from the traditional smooth ones, as shown below. Therefore, the UWM indeed unifies the smooth waves and the peaked solitary waves as a whole, for the first time, to the best of the author's knowledge.

\subsection{Peaked solitary waves by the linear UWM}

As mentioned in $\S 2$, both of the base functions (17) and (26) automatically satisfy the Laplace equation (3) in the domain $0<x<+\infty$, the bottom condition (6) and the bounded condition (12). In the frame of the linear UWM, the former satisfies the periodic boundary condition (8) and gives the traditional Airy waves, which are infinitely differentiable everywhere, as mentioned in $\S 3$. The latter satisfies the decay condition (9) and gives peaked solitary waves, as shown below.

In the interval $0<x<+\infty$, we have the velocity potential in the form

$$
\phi^{+}(x, z)=\alpha A \cos [k(z+1)] e^{-k x}, \quad 0<x<+\infty,
$$

where the superscript + denotes a solution in the interval $x \in(0,+\infty), \alpha$ denotes the (dimensionless) phase speed, $A$ is a constant related to the wave height, and $k>0$ is a parameter related to the phase speed $\alpha$, respectively. Note that the above expression automatically satisfies the Laplace equation (3) in the domain $0<x<+\infty$, the bottom condition (6), the decay condition (9) and the bounded condition (12). Substituting (27) into the linear boundary condition (15) gives

$$
\alpha k A\left(\alpha^{2} k \cos k-\sin k\right) \exp (-k x)=0, \quad 0<x<+\infty,
$$


which leads to the important relationship

$$
\alpha^{2}=\frac{\tan k}{k}, \quad n \pi<k<n \pi+\frac{\pi}{2}
$$

where $n \geq 0$ is an integer. Similarly, defining $\phi^{-}$the potential function in the interval $x \in(-\infty, 0)$, we gain exactly the same result. Obviously, we have $\alpha \geq 1$, i.e. $c \geq \sqrt{g D}$. This is different from the periodic Airy wave whose phase speed has the property $c \leq \sqrt{g D}$.

Given the dimensionless phase velocity $\alpha$, the transcendental equation (28) has an infinite number of solutions $k_{n}$, where

$$
n \pi<k_{n}<n \pi+\frac{\pi}{2}, \quad n \geq 0 .
$$

For the sake of simplicity, define the set

$$
\mathbf{K}_{\alpha}=\left\{k_{n}: \alpha^{2}=\frac{\tan k_{n}}{k_{n}}, n \pi<k_{n}<n \pi+\frac{\pi}{2}, n=0,1,2,3, \cdots\right\} .
$$

Write $k_{\nu} \in \mathbf{K}_{\alpha}$, where $\nu \geq 0$ is an integer. Then, we have the solution

$$
\phi^{+}(x, z)=\alpha A_{\nu} \cos \left[k_{\nu}(z+1)\right] \exp \left(-k_{\nu} x\right), \quad 0<x<+\infty .
$$

According to the linearized boundary condition (16), the corresponding elevation of the solitary wave reads

$$
\begin{aligned}
\zeta^{+}(x) & =\left.\alpha \frac{\partial \phi^{+}}{\partial x}\right|_{z=0}=-\alpha^{2} A_{\nu} k_{\nu} \cos k_{\nu} \exp \left(-k_{\nu} x\right) \\
& =-A_{\nu} \sin k_{\nu} \exp \left(-k_{\nu} x\right), \quad 0<x<+\infty
\end{aligned}
$$

where $\alpha^{2} k_{\nu} \cos k_{\nu}=\sin k_{\nu}$ given by (28) is applied. Due to the continuity of wave elevation, we have at $x=0$ the wave elevation

$$
\zeta(0)=\lim _{x \rightarrow 0} \zeta^{+}(x)
$$

Then, according to (11), we have the relation

$$
H_{w}=-A_{\nu} \sin k_{\nu}
$$

Thus, using the symmetry (13), we gain a peaked solitary wave

$$
\zeta(x)=H_{w} e^{-k_{\nu}|x|}, \quad-\infty<x<+\infty .
$$

This is a solitary wave that seriously has only one crest, but with a discontinuous 1st-derivative $\zeta^{\prime}(x)$ at crest! For example, in the case of $\alpha=3^{3 / 4} / \sqrt{\pi}$, we have an infinite number of peaked solitary waves with $k_{0}=\pi / 3, k_{1}=4.58117$, and so on: the corresponding elevations of the peaked solitary waves are as shown in Fig. 1, respectively. Note that all of them have a peaked crest. Besides, the larger the value 


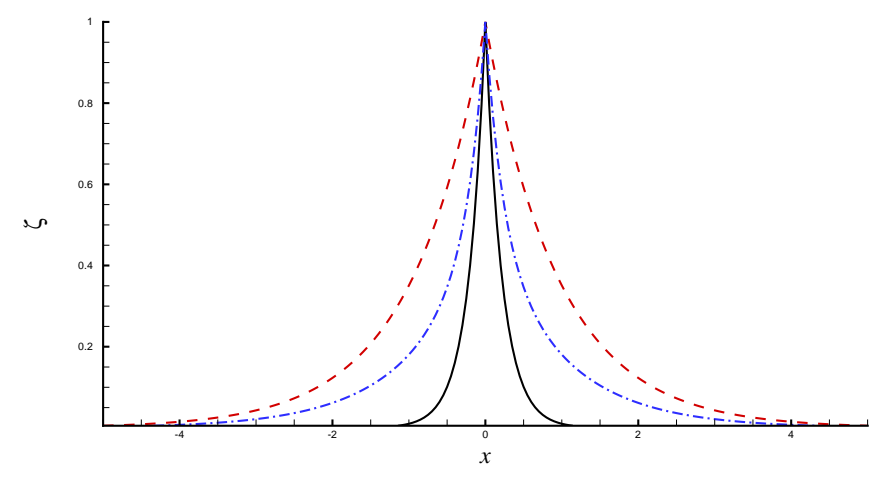

Figure 1: $\zeta(x) / H_{w}$ in the case of $k_{0}=\pi / 3, k_{1}=4.58117$ with $\alpha=3^{3 / 4} / \sqrt{\pi}$. Dashed line: $\exp \left(-k_{0}|x|\right)$; Solid line: $\exp \left(-k_{1}|x|\right)$; Dash-dotted line: $\left[\exp \left(-k_{0}|x|\right)+\right.$ $\left.\exp \left(-k_{1}|x|\right)\right] / 2$.

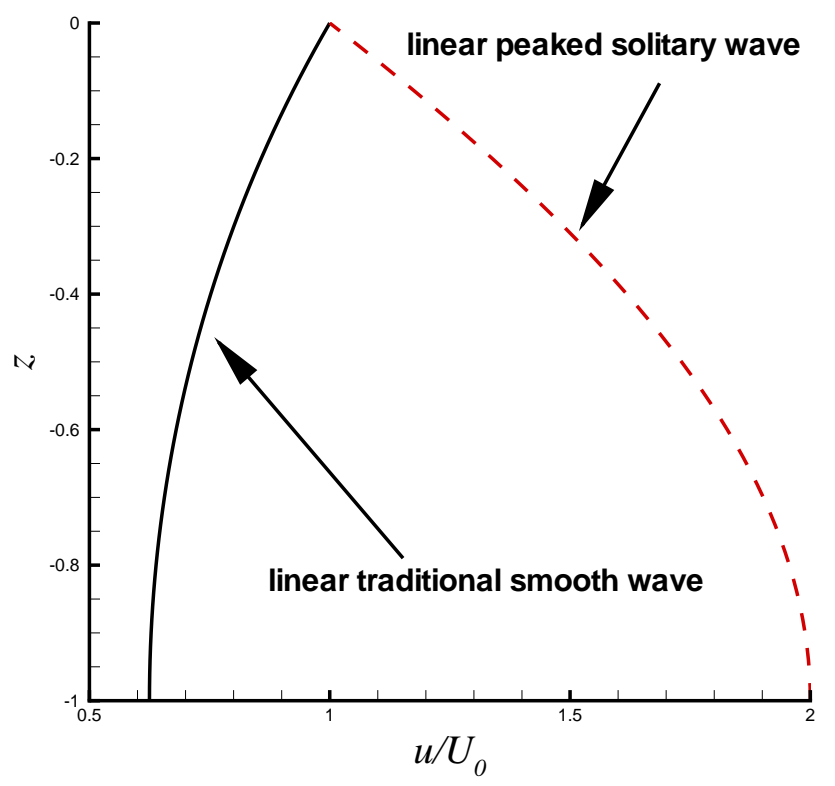

Figure 2: Velocity profile $u / U_{0}$ at $x=0$ in the case of $k_{0}=\pi / 3$ with $U_{0}=H_{w} / \alpha$. Solid line: periodic Airy wave; Dashed line: linear peaked solitary wave. 
of $k_{\nu}$, the sharper the peaked solitary wave. These are essentially different from the traditional periodic and solitary progressive waves that are infinitely differentiable everywhere. This clearly indicates the novelty of the peaked solitary waves given by the UWM. It should be emphasized that (35) is exactly the same as the famous peaked solitary wave (2) of the $\mathrm{CH}$ equation (1) when $H_{w}=k_{\nu}=c$, with the definition $x=X-c t$.

In the interval $0<x<+\infty$, the corresponding horizontal velocity reads

$$
u^{+}(x, z)=\frac{\partial \phi^{+}}{\partial x}=\frac{\alpha k_{\nu} H_{w} \cos \left[k_{\nu}(z+1)\right] e^{-k_{\nu} x}}{\sin \left(k_{\nu}\right)} .
$$

Using the symmetry (13), we have

$$
u^{-}(x, z)=u^{+}(-x, z)=\frac{\alpha k_{\nu} H_{w} \cos \left[k_{\nu}(z+1)\right] e^{k_{\nu} x}}{\sin \left(k_{\nu}\right)}, \quad-\infty<x<0 .
$$

At $x=0, u$ is continuous and we have the corresponding horizontal velocity

$$
U(z)=\lim _{x \rightarrow 0} \frac{\partial \phi^{+}}{\partial x}=\frac{\alpha k_{\nu} H_{w} \cos \left[k_{\nu}(z+1)\right]}{\sin \left(k_{\nu}\right)} .
$$

Thus, in the whole domain $-\infty<x<+\infty$, we have a uniform expression

$$
\frac{u}{U_{0}}=\frac{\cos \left[k_{\nu}(z+1)\right] e^{-k_{\nu}|x|}}{\cos \left(k_{\nu}\right)}, \quad x \in(-\infty,+\infty),
$$

where $U_{0}=H_{w} / \alpha$. Thus, as long as the horizontal velocity at $x=0$ is given by the above expression, the peaked solitary wave with the symmetry and the permanent surface form exists! Note that, for given $x$, the horizontal velocity $u$ of the peaked solitary wave increases as $z$ varies from the free surface $(z=0)$ to the bottom $(z=$ $-1)$ : in other words, $u$ on the bottom is always larger than that on the free surface. For example, when $k_{0}=\pi / 3$ (corresponding to the phase speed $c=3^{3 / 4} / \sqrt{\pi}$ ), the horizontal velocity on the bottom is always twice of that on the free surface, as shown in Fig. 2. This is quite different from the traditional smooth waves whose horizontal velocity $u$ on bottom is always less than that on free surface. This indicates once again the novelty of the peaked solitary waves given by the UWM.

Let $v^{+}(x, z)$ and $v^{-}(x, z)$ denote the vertical velocity in the interval $x>0$ and $x<0$, respectively. In the domain $0<x<+\infty$, the corresponding vertical velocity reads

$$
v^{+}(x, z)=\frac{\partial \phi^{+}}{\partial z}=\frac{\alpha k_{\nu} H_{w} \sin \left[k_{\nu}(z+1)\right] e^{-k_{\nu} x}}{\sin k_{\nu}} .
$$

Using the symmetry (13), we have

$$
v^{-}(x, z)=-v^{+}(-x, z)=-\frac{\alpha k_{\nu} H_{w} \sin \left[k_{\nu}(z+1)\right] e^{k_{\nu} x}}{\sin k_{\nu}}, \quad-\infty<x<0 .
$$



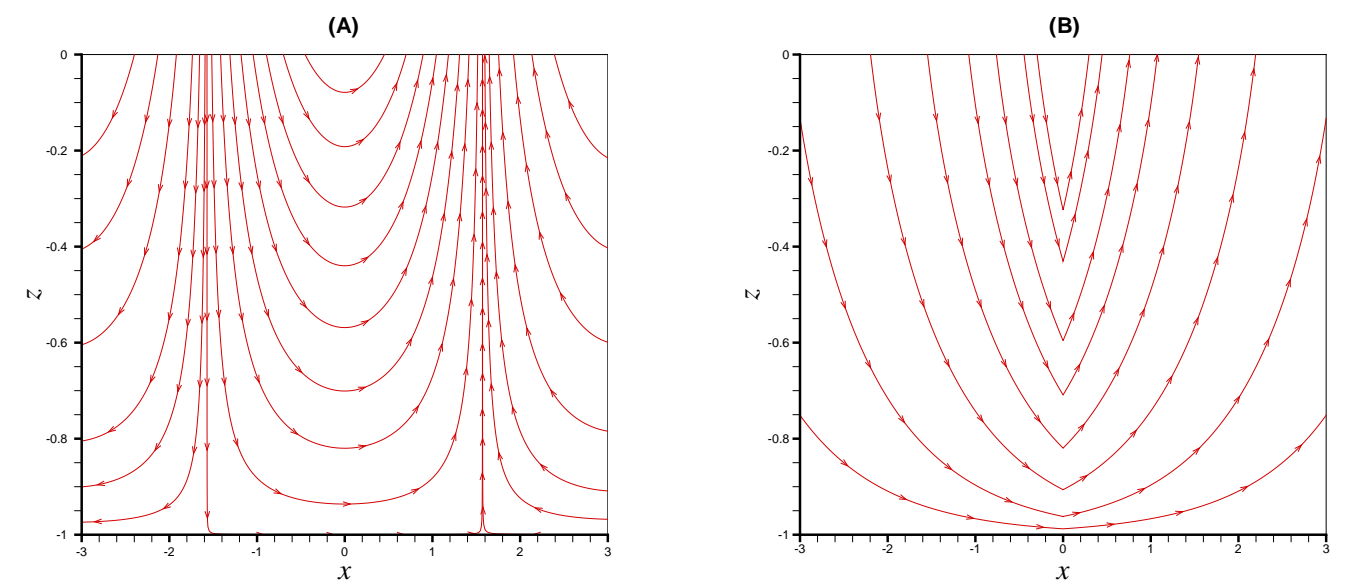

Figure 3: Comparison of streamlines given by the stream-function $\psi /\left(\alpha H_{w}\right)$ in case of $k=1$. (A): Airy wave; (B): peaked solitary wave given by the linear UWM.

As $x \rightarrow 0$, we have the limits

$$
\lim _{x \rightarrow 0} v^{+}=\frac{\alpha k_{\nu} H_{w}}{\sin k_{\nu}} \sin \left[k_{\nu}(z+1)\right], \quad \lim _{x \rightarrow 0} v^{-}=-\frac{\alpha k_{\nu} H_{w}}{\sin k_{\nu}} \sin \left[k_{\nu}(z+1)\right]
$$

so that

$$
\lim _{x \rightarrow 0} v^{+}=-\lim _{x \rightarrow 0} v^{-} \neq 0 .
$$

However, according to the boundary condition (14), the vertical velocity $v$ exactly equals to zero at $x=0$, i.e. $v(0, z)=0$. Thus, at the interface $x=0$, the vertical velocity $v$ is discontinuous: $v$ changes sign as we cross the interface $x=0$. Besides, for given $z$, the jump of $v$ at $x=0$, i.e.

$$
\lim _{x \rightarrow 0}\left(v^{+}-v^{-}\right)=2 \lim _{x \rightarrow 0} v^{+}
$$

is directly proportional to $k_{\nu}$.

Is such kind of discontinuity of the vertical velocity acceptable in the frame of inviscid fluid? It should be emphasized here that, in the frame of the traditional theories for smooth waves, there exist the similar discontinuity and jump of velocity on an interface, which however are widely accepted by the scientific community. For instance, on the free surface of interfacial waves [34-37], although the velocity normal to the interface is continuous, the tangential velocity is discontinuous, as pointed out by Lamb [38] ( $\$ 231$, page 371): "the tangential velocity changes sign as we cross the surface", but "in reality the discontinuity, if it could ever be originated, would be immediately abolished by viscosity". Similarly, in the frame of the inviscid fluid, the peaked solitary waves should be acceptable, too. We will discuss this problem later in details.

The stream function of the peaked solitary wave reads

$$
\psi(x, z)=\frac{\alpha H_{w} \sin \left[k_{\nu}(z+1)\right] e^{-k_{\nu}|x|}}{\sin \left(k_{\nu}\right)}, \quad|x| \in(0,+\infty) .
$$


For the linear Airy waves, we have the stream function

$$
\psi(x, z)=\frac{\alpha H_{w} \sinh [k(1+z)] \cos (k x)}{2 \sinh (k)}, \quad x \in(-\infty,+\infty) .
$$

The streamlines for Airy wave are smooth everywhere and periodic in the horizontal direction, as shown in Fig. 3. However, the streamlines of the peaked solitary waves are neither smooth at crest $(x=0)$ nor periodic in the horizontal direction. Note that the velocity field of the traditional Airy waves is also smooth everywhere, but there exists the discontinuity of the velocity at crest $x=0$ for the peaked solitary waves. This illustrates once again that the peaked solitary waves are indeed quite different from the traditional smooth ones.

Mathematically speaking, given a dimensionless phase velocity $\alpha$, there exist an infinite number of $k_{\nu}$ satisfying (28), and each of them corresponds to a solution of the linear UWM. This is quite different from the smooth periodic progressive Airy wave $\zeta(x)=A \cos (k x)$ that is unique for a given $\alpha$. This is because, given a dimensionless phase speed $\alpha$, the transcendental equation (19) has an unique solution, but the transcendental equation (28) has an infinite number of solutions! Note that, unlike the smooth Airy wave theory, the parameter $k_{\nu}$ of the peaked solitary wave does not denote the wave number, but the decay-rate of the wave elevation as $x \rightarrow+\infty$ : the larger the value of $k_{\nu}$, more quickly the wave elevation decays to zero. When the wave height is fixed, define the decay length

$$
\lambda_{a}=2\left|x_{B}\right|
$$

of the peaked solitary waves, where $x_{B}$ is determined by the nonlinear equation $\zeta\left(x_{B}\right)=10^{-4}$. Essentially, for a fixed wave height, we can regard the decay length $\lambda_{a}$ as a characteristic length of peaked solitary waves in the horizontal direction, called the "actual wavelength", although in theory wavelength of a solitary waves is infinite. In this meaning, for a fixed wave height, the peaked solitary waves are dispersive with the actual wavelength $\lambda_{a}$. Besides, according to (28), the peaked solitary wave can propagate very quickly even if the wave height $H_{w}$ is small, since $\tan k_{\nu} / k_{\nu} \rightarrow+\infty$ as $k_{\nu} \rightarrow \nu \pi+\pi / 2$, where $k_{\nu} \in \mathbf{K}_{\alpha}$ and $\nu \geq 0$ is an integer.

The kinetic energy of the smooth progressive Airy waves given by the linear UWM reads

$$
E_{k}=\frac{1}{2}\left(u^{2}+v^{2}\right)=\left[\frac{\alpha H_{w} k}{4 \sinh (k)}\right]^{2}\{\cosh [2 k(1+z)]+\cos (2 k x)\},
$$

which is periodic in the horizontal direction and decreases exponentially as $z$ decreases from the free surface $(z=0)$ to the bottom $(z=-1)$. However, according to (36) and (401) or (41), we have the kinetic energy of the peaked solitary waves given by the linear UWM:

$$
E_{k_{\nu}}=\frac{1}{2}\left(u^{2}+v^{2}\right)=\frac{\left(\alpha H_{w} k_{\nu}\right)^{2}}{2 \sin ^{2}\left(k_{\nu}\right)} \exp \left(-2 k_{\nu}|x|\right),
$$

which is independent of $z$ and decays exponentially as $|x|$ increases. This is quite different from the traditional smooth Airy waves. It illustrates once again the novelty of the peaked solitary waves given by the UWM. 
Since the governing equation (3), the two linearized free surface conditions (15) (16) and all other conditions are linear, the solution of the peaked solitary wave can be expressed in a general form

$$
\phi^{+}(x, z)=\alpha \sum_{n=0}^{+\infty} A_{n} \cos \left[k_{n}(z+1)\right] \exp \left(-k_{n} x\right), \quad 0<x<+\infty .
$$

Using the linear boundary condition (16), we have the corresponding elevation of the solitary wave

$$
\begin{aligned}
\zeta^{+}(x) & =\left.\alpha \frac{\partial \phi^{+}}{\partial x}\right|_{z=0}=-\alpha^{2} \sum_{n=0}^{+\infty} A_{n} k_{n} \cos k_{n} \exp \left(-k_{n} x\right) \\
& =-\sum_{n=0}^{+\infty} A_{n} \sin k_{n} \exp \left(-k_{n} x\right) \\
& =\sum_{n=0}^{+\infty} b_{n} \exp \left(-k_{n} x\right), \quad 0<x<+\infty
\end{aligned}
$$

where the relationship (28) is applied. Using the symmetry (13), we have

$$
\begin{aligned}
\zeta(x) & =\sum_{n=0}^{+\infty} b_{n} \exp \left(-k_{n}|x|\right), \\
u(x, z) & =-\alpha \sum_{n=0}^{+\infty} A_{n} k_{n} \cos \left[k_{n}(z+1)\right] \exp \left(-k_{n}|x|\right), \\
v^{+}(x, z) & =-\alpha \sum_{n=0}^{+\infty} A_{n} k_{n} \sin \left[k_{n}(z+1)\right] \exp \left(-k_{n} x\right), \\
v^{-}(x, z) & =\alpha \sum_{n=0}^{+\infty} A_{n} k_{n} \sin \left[k_{n}(z+1)\right] \exp \left(k_{n} x\right)
\end{aligned}
$$

where the superscripts + and - denote the interval $x \in(0,+\infty)$ and $x \in(-\infty, 0)$, respectively. Note that, using the restriction (11), we have a linear algebraic equation

$$
H_{w}=\sum_{n=0}^{+\infty} b_{n}
$$

which has an infinite number of solutions of $b_{n}$ for a given value of $H_{w}$, where $n \geq 0$ is an integer. In other words, from mathematical viewpoints, given a wave height $H_{w}$ and a phase speed $\alpha \geq 1$, there exist an infinite number of peaked solitary waves. This is completely different from the smooth periodic progressive Airy wave that is unique for given phase speed and wave height. This is because, given a phase speed $\alpha$, the transcendental equation (19) has an unique solution but (28) has an infinite number of ones! Thus, different peaked solitary waves might have exactly the same 
phase speed. For example, in the case of $k_{0}=\pi / 3, k_{1}=4.58117$ when $\alpha=3^{3 / 4} / \sqrt{\pi}$, the peaked solitary wave

$$
\zeta(x)=\frac{1}{2}\left(e^{-k_{0}|x|}+e^{-k_{1}|x|}\right)
$$

is different from the peaked solitary waves $\exp \left(-k_{0}|x|\right)$ and $\exp \left(-k_{1}|x|\right)$ but has the same phase speed with them, as shown in Fig 1. Note that, in the frame of the linear UWM, the crest of each peaked solitary wave may be at different position. So, the peaked wave elevation can be generally expressed by

$$
\zeta(x)=\sum_{n=0}^{+\infty} b_{n} \exp \left(-k_{n}\left|x-\xi_{0}\right|\right),
$$

where $b_{n}$ and $\xi_{n}$ are real numbers.

Like traditional smooth Airy's wave, since the elevation (35) of the peaked solitary wave is gained by the linear wave equations, the value of $H_{w}$ can be negative, corresponding to a peaked solitary wave in the form of depression. For example, $\zeta(x)=-\exp (-|x|) / 10$ is a peaked solitary wave of depression. Such kind of peaked solitary waves have been never reported for surface waves. This once again indicates the novelty of the peaked solitary waves given by the UWM.

It is traditionally believed that solitary waves are always governed by nonlinear differential equations and exist in shallow water. However, we illustrate here that solitary waves may exist even in the frame of the linear wave equations in water of finite depth. It should be emphasized that the peaked solitary wave (35) includes the famous peaked solitary wave (2) found by Casamma \& Holm [6]. This might reveal the origin of the peaked solitary waves of the $\mathrm{CH}$ equation (1), since the $\mathrm{CH}$ equation was derived from the fully nonlinear wave equations under some assumptions in shallow water. Note that the peaked solitary wave (35) is valid not only in shallow water but also in water of finite depth, and thus is more general. Logically speaking, the peaked solitary wave (35) is as acceptable as the peaked solitary waves (2) of the $\mathrm{CH}$ equation (1), as shown later in more details.

In summary, the linear UWM admits not only the traditional progressive waves with smooth crest, but also the peaked solitary waves that include the famous peaked solitary wave (2) given by the $\mathrm{CH}$ equation (11) and besides have many unusual characteristics. Thus, the UWM unifies, for the first time, the traditional smooth waves and the peaked solitary waves. In other words, the linear peaked solitary waves are consistent with the traditional smooth ones, and thus are as acceptable as the traditional ones in the frame of inviscid fluid. It is found that the linear peaked solitary waves have many unusual characteristics that are quite different from those of the traditional smooth ones. First, its 1st-derivative of elevation is discontinuous at crest with a discontinuous vertical velocity. Besides, the phase speed of the peaked solitary waves has nothing to do with wave height: in fact, it is dispersive (when wave height is fixed) with the decay length, i.e. the so-called "actual wavelength" $\lambda_{a}$. In addition, unlike the traditional smooth waves whose kinetic energy is periodic in the horizontal direction and decreases exponentially as $z$ decreases from free surface to bottom, the 
kinetic energy of the linear peaked solitary waves decreases in the horizontal direction as $|x|$ increases, but keeps the same from free surface to bottom, i.e. it is independent of $z$ at all. All of these reveal the novelty of the linear peaked solitary waves.

In the following section, we further check and confirm these unusual characteristics of the linear peaked solitary waves using the exact UWM based on the symmetry and the fully nonlinear wave equations.

\subsection{Peaked solitary waves by the nonlinear UWM}

As shown in $\S 4.1$, the peaked solitary surface waves given by the linear UWM with the free surface conditions (15) and (16) have some unusual characteristics that are quite different from the smooth periodic and solitary ones. Does the exact UWM based on the symmetry (13) and the fully nonlinear wave equations (3) - (12) indeed admit such kind of peaked solitary waves? Do this kind of peaked solitary waves have the same unusual characteristics as those reported in $\S 4.1$ ?

To answer these questions, we consider here the solitary surface waves with a finite wave height in a finite water depth so that the nonlinear terms of the free surface conditions (4) and (5) are not negligible, and besides $z=0$ is not a good approximation of the free surface $\zeta(x)$. In other words, we had to accurately solve the fully nonlinear wave equations (31) - (12) in the interval $0<x<+\infty$ with the symmetry (13).

In this section, an analytic approximation technique for highly nonlinear differential equations proposed by Liao, namely the homotopy analysis method (HAM) [14 20, is applied to solve the exact UWM based on the symmetry (13) and the fully nonlinear wave equations (3) - (12). Unlike perturbation techniques, the HAM does not need any assumptions of small/large physical parameters, since it is based on the homotopy, a basic concept in topology. Besides, the HAM provides us great freedom to choose base functions and equation-type for high-order approximation equations. Especially, by means of the so-called "convergence-control parameter" that has no physical meanings, the HAM provides us a convenient way to guarantee the convergence of approximation series. In essence, it is the so-called "convergence-control parameter" that differs the HAM from all other analytic approximation techniques, as pointed out by Liao [19]. Therefore, the HAM is valid for highly nonlinear problems, as shown by lots of successful applications in fluid mechanics, applied mathematics, physics and finance. For example, by means of the HAM, Liao [15] gained, for the first time, convergent series solution of Blasius and Falker-Skan boundary-layer flows, which are uniformly valid in the whole field of flow (Note that the traditional power series given by Blasius [39] is valid only in the near field, and thus had to be matched with another asymptotic approximation of flow in far field). Besides, using the HAM as a tool, the exact Navier-Stokes equations were solved by Turkyilmazoglu [40] for a compressible boundary layer flow due to a porous rotating disk, and by $\mathrm{Xu}$ et al. [41. for viscous flows in a porous channel with orthogonally moving walls. Furthermore, the limit cycle of Duffing - van der Pol equation was solved by Turkyilmazoglu [42], 
and the two coupled Van der Pol equations were solved by Li et al. [43]. Especially, by means of the HAM, some new boundary layer flows have been found by Liao [44] and by Liao \& Magyari [45, which have been neglected by other analytic and even numerical techniques. In addition, the HAM has been successfully applied to solve some nonlinear PDEs with moving boundary conditions, such as those about the famous problem of the American put option in finance. For example, Zhu [46] successfully applied the HAM to give a series approximation of the American put option and gained the optimal exercise boundary valid for a couple of years, while perturbative and/or asymptotic formulas are accurate only in a few days or weeks. All of these illustrate the potential and validity of the HAM for highly nonlinear problems.

It should be emphasized that the HAM has been successfully applied to solve the fully nonlinear wave equations, too. Using the traditional base functions (17), Liao \& Cheung [47] applied the HAM to solve the smooth periodic progressive surface waves in deep water and obtained convergent solutions for waves with large amplitude even close to the limiting case. Their analytic results agree quite well with those given by Schwartz [48] and Longuet-Higgins [49]. Besides, using the same base functions (17), Tao et al. [50] successfully applied the HAM to solve the fully nonlinear wave equations for smooth periodic progressive waves in finite water depth, and their analytic results agree well not only with the analytic ones given by Cokelet [21] and Fenton [51] but also with the experimental ones reported by Mehaute et al. 52. Currently, $\mathrm{Xu}$ et al. [53] successfully applied the HAM to investigate the steady-state fullyresonant progressive waves in finite water depth, and found, for the first time, the multiple steady-state resonant waves whose wave spectrums are independent of time, i.e. without exchange of wave energy. All of these demonstrate the validity of the HAM for the fully nonlinear wave equations (3) - (12).

\subsubsection{Analytic approach based on the HAM}

In short, the fully nonlinear wave equations (3) - (12) in the domain $0<x<+\infty$ can be solved by means of the HAM and the evanescent base functions (26) in a rather similar way as those by Liao \& Cheung [47] and Tao et al. [50], although they used the traditional base functions (17) with perfect smoothness. Then, we expand these results to the whole domain $x \in(-\infty,+\infty)$ using the symmetry (13). In this way, we can gain the peaked solitary waves in the whole domain $x \in(-\infty,+\infty)$ by means of the UWM.

Due to the symmetry (13), we need consider the case $x>0$ only. As mentioned in $\S 4.1$ for the linearized UWM, given a phase speed $\alpha$, there exists an infinite number of peaked solitary waves $\zeta(x)=H_{w} \exp \left(-k_{\nu} x\right)$ with different decay-parameter $k_{\nu}$ satisfying the transcendental equation (28), where $k_{\nu} \geq 0$ is an integer. So, the general expression of the peaked solitary waves becomes rather complicated when the nonlinear boundary conditions are considered. Thus, for the sake of simplicity, we consider here only the peaked solitary waves with the primary decay-parameter $0 \leq k_{0} \leq \pi / 2$. From now on, let $k$ denote the primary decay-parameter $k_{0}$, if not mentioned. Obvi-

ously, due to the nonlinearity of the two free surface conditions, the peaked solitary 
wave elevation should contain the terms $\exp (-n k x)$, and correspondingly the velocity potential function $\phi(x, z)$ should be expressed in the form

$$
\phi(x, z)=\sum_{n=1}^{+\infty} a_{n} \cos [n k(z+1)] \exp (-n k x), \quad x>0, k>0
$$

which automatically satisfies the Lapalce equation (3) in the domain $0<x<+\infty$, the bottom condition (6) and the bounded condition (12), where $k=k_{0} \in \mathbf{K}_{\alpha}$ defined by (30) is a primary decay-parameter, and $a_{n}$ is a constant to be determined. We search for the solitary surface waves in the form

$$
\zeta(x)=\sum_{n=1}^{+\infty} b_{n} \exp (-n k x), \quad x>0, k=k_{0} \in \mathbf{K}_{\alpha},
$$

where $b_{n}$ is a constant coefficient to be determined. The above expressions (157) and (58) provide us the so-called solution-expression of $\phi(x, z)$ and $\zeta(x)$, respectively, which play important role in the frame of the HAM, as shown below.

Let $\phi_{0}(x, z), \zeta_{0}(x)$ denote the initial guess of the velocity potential $\phi(x, z)$ and the wave elevation $\zeta(x)$ in the interval $x \in(0,+\infty)$, respectively. To apply the HAM, we should first of all construct two continuous variations from the initial guess $\phi_{0}(x, z), \zeta_{0}(x)$ to the exact solution $\phi(x, z), \zeta(x)$, respectively. This can be easily done by means of the homotopy, a basic concept in topology, as shown below.

First, according to the solution expression (57), we choose

$$
\phi_{0}(x, z)=A_{0} \cos [k(z+1)] e^{-k x}, \quad x>0, k>0,
$$

as the initial guess of the velocity potential $\phi(x, z)$, where $A_{0}$ is a constant to be determined. Note that, different from Liao \& Cheung [47] and Tao et al. [50], the evanescent-mode base-function (26) is used here. Note also that $\phi_{0}(x, z)$ automatically satisfies the Laplace equation (3) in the interval $0<x<+\infty$, the bottom condition (6) and the bounded condition (12). Besides, following Liao \& Cheung [47] and Tao et al. [50], we choose

$$
\zeta_{0}(x)=0
$$

as the initial guess of wave elevation $\zeta(x)$.

Secondly, according to (4), we define a nonlinear operator

$$
\mathcal{N} \phi=\alpha^{2} \frac{\partial^{2} \phi}{\partial x^{2}}+\frac{\partial \phi}{\partial z}-\alpha \frac{\partial}{\partial x}(\nabla \phi \cdot \nabla \phi)+\nabla \phi \cdot \nabla\left(\frac{1}{2} \nabla \phi \cdot \nabla \phi\right) .
$$

Let $q \in[0,1]$ denote an embedding parameter, $c_{\phi}$ and $c_{\eta}$ be two non-zero auxiliary parameters without physical meanings, called the convergence-control parameters, and $\mathcal{L}$ denote an auxiliary linear operator, respectively. Following Liao \& Cheung [47] and Tao et al. [50, we construct the so-called zeroth-order deformation equation

$$
\nabla^{2} \Phi(x, z ; q)=0, \quad 0<x<+\infty, \quad z \leq \eta(x ; q)
$$


subject to the boundary conditions on the unknown free surface $z=\eta(x ; q)$ :

$$
\begin{array}{ll}
(1-q) \mathcal{L}\left[\Phi(x, z ; q)-\phi_{0}(x, z)\right]=c_{\phi} q \mathcal{N}[\Phi(x, z ; q)], & 0<x<+\infty \\
(1-q) \eta(x ; q)=c_{\eta} q\left[\eta(x ; q)-\alpha \frac{\partial \Phi}{\partial x}+\frac{1}{2} \nabla \Phi \cdot \nabla \Phi\right], & 0<x<+\infty
\end{array}
$$

and the boundary condition at the bottom

$$
\frac{\partial \Phi}{\partial z}=0, \quad z=-1, \quad 0<x<+\infty
$$

If the wave height $H_{w}$ is given, there exists the additional condition:

$$
\lim _{x \rightarrow 0} \eta(x ; q)=H_{w}
$$

Note that $\Phi(x, z ; q)$ and $\eta(x ; q)$ depend not only on the original physical variables $x, z$ but also on the embedding parameter $q \in[0,1]$ and the two convergence-control parameter $c_{\phi}, c_{\eta}$, though $q, c_{\phi}$ and $c_{\eta}$ have no physical meanings at all. It should be emphasized that we have great freedom to choose the values of the convergence-control parameters $c_{\phi}$ and $c_{\eta}$. Following Liao \& Cheung [47] and Tao et al. [50], we choose the auxiliary linear operator

$$
\mathcal{L} \phi=\alpha^{2} \frac{\partial^{2} \phi}{\partial x^{2}}+\frac{\partial \phi}{\partial z}
$$

which has the property $\mathcal{L}[0]=0$. Note that $\mathcal{L}$ is exactly the linear part of the nonlinear operator $\mathcal{N}$ defined by (61). In this way, the zeroth-order deformation equations (62) - (66) are well defined.

When $q=0$, we have from (64) that

$$
\eta(x ; 0)=0=\zeta_{0}(x)
$$

and then the corresponding zeroth-order deformation equations become

$$
\nabla^{2} \Phi(x, z ; 0)=0, \quad z \leq 0, \quad 0<x<+\infty,
$$

subject to the boundary conditions on the known free surface

$$
\mathcal{L}\left[\Phi(x, z ; 0)-\phi_{0}(x, z)\right]=0, \quad \text { when } z=0, \quad 0<x<+\infty,
$$

and the boundary condition at the bottom

$$
\frac{\partial \Phi(x, z ; 0)}{\partial z}=0, \quad z=-1, \quad 0<x<+\infty .
$$

Since the auxiliary linear operator $\mathcal{L}$ has the property $\mathcal{L}[0]=0$ and besides the initial guess $\phi_{0}(x, z)$ defined by (159) satisfies the Laplace equation (3) and the bottom condition ([6), it is straightforward that

$$
\Phi(x, z ; 0)=\phi_{0}(x, z)
$$


When $q=1$, since $c_{\phi} \neq 0$ and $c_{\eta} \neq 0$, the zeroth-order deformation equations (62) - (66) are equivalent to the original, fully nonlinear wave equations (3) - (11), respectively, so that we have the relationship

$$
\Phi(x, z ; 1)=\phi(x, z), \quad \eta(x ; 1)=\zeta(x) .
$$

Thus, as the embedding parameter $q$ increases from 0 to $1, \Phi(x, z ; q)$ and $\eta(x ; q)$ indeed vary continuously from the initial guess $\phi_{0}(x, z), \zeta_{0}(x)$ to the exact solution $\phi(x, z), \zeta(x)$ of the fully nonlinear wave equations (3) - (11), respectively. Therefore, the zeroth-order deformation equations (62) - (66) truly construct such a kind of continuous variation that provides a base of our analytic approach, as shown below.

Since both of $\Phi(x, z ; q)$ and $\eta(x ; q)$ are dependent upon the embedding parameter $q \in[0,1]$, we can expand them in Maclaurin series with respect to $q$ to gain the so-called homotopy-Maclaurin series

$$
\begin{aligned}
\Phi(x, z ; q) & =\phi_{0}(x, z)+\sum_{m=1}^{+\infty} \phi_{m}(x, z) q^{m} \\
\eta(x ; q) & =\sum_{m=1}^{+\infty} \zeta_{m}(x) q^{m}
\end{aligned}
$$

where

$$
\phi_{m}(x, z)=\left.\frac{1}{m !} \frac{\partial^{m} \Phi(x, z ; q)}{\partial q^{m}}\right|_{q=0}, \quad \zeta_{m}(x)=\left.\frac{1}{m !} \frac{\partial^{m} \eta(x ; q)}{\partial q^{m}}\right|_{q=0}
$$

and the relationship (68) and (72) are used. However, it is well known that a Maclaurin series often has a finite radius of convergence. Fortunately, both of $\Phi(x, z ; q)$ and $\eta(x ; q)$ contain the two convergence-control parameters $c_{\phi}$ and $c_{\eta}$, which have great influence on the convergence of the Maclaurin series of $\Phi(x, z ; q)$ and $\eta(x ; q)$, as shown by Liao \& Cheung [47] and Tao et al. [50]. Here, it should be emphasized once again that we have great freedom to choose the values of $c_{\phi}$ and $c_{\eta}$. Thus, if the convergence-control parameters $c_{\phi}$ and $c_{\eta}$ are properly chosen so that the above homotopy-Maclaurin series are convergent at $q=1$, we have the homotopy-series solution

$$
\begin{aligned}
\phi(x, z) & =\phi_{0}(x, z)+\sum_{m=1}^{+\infty} \phi_{m}(x, z) \\
\zeta(x) & =\sum_{m=1}^{+\infty} \zeta_{m}(x) .
\end{aligned}
$$

The equations for the unknown $\phi_{m}(x, z)$ and $\zeta_{m}(x)$ can be derived directly from the zeroth-order deformation equations. Like Liao \& Cheung [47] and Tao et al. [50], substituting the series (74) and (75) into the zeroth-order deformation equations (62) - (66), then equating the like-power of $q$, we gain

$$
\zeta_{m}(x)=\left.\left\{c_{\eta} \Delta_{m-1}^{\eta}+\chi_{m} \zeta_{m-1}\right\}\right|_{z=0}, \quad m \geq 1, \quad 0<x<+\infty,
$$


where

$$
\Delta_{m}^{\eta}=\zeta_{m}-\alpha \bar{\phi}_{m, 1}+\Gamma_{m, 0}
$$

and the $m$ th-order deformation equation

$$
\nabla^{2} \phi_{m}(x, z)=0, \quad m \geq 1, z \leq 0, \quad 0<x<+\infty
$$

subject to the boundary condition on the known free surface $z=0$ :

$$
\overline{\mathcal{L}}\left(\phi_{m}\right)=\left.\left(\alpha^{2} \frac{\partial^{2} \phi_{m}}{\partial x^{2}}+\frac{\partial \phi_{m}}{\partial z}\right)\right|_{z=0}=R_{m}(x), \quad 0<x<+\infty,
$$

and the bottom condition

$$
\frac{\partial \phi_{m}}{\partial z}=0, \quad z=-1, \quad 0<x<+\infty
$$

where

$$
\begin{aligned}
R_{m}(x) & =\left.\left\{c_{\phi} \Delta_{m-1}^{\phi}+\chi_{m} S_{m-1}-\bar{S}_{m}\right\}\right|_{z=0}, 0<x<+\infty \\
\chi_{n} & = \begin{cases}0, & \text { when } n \leq 1 \\
1, & \text { when } n>1 .\end{cases}
\end{aligned}
$$

The detailed derivations of $\Delta_{m-1}^{\eta}, \Delta_{m-1}^{\phi}, S_{m-1}, \bar{S}_{m}$ with all related formulas are given explicitly in the Appendix. These formulas are essentially the same as those for the smooth periodic progressive waves used by Liao \& Cheung [47] and Tao et al. [50], although we explicitly give all formulas in details so that high-order approximations can be gained more efficiently.

Note that the dimensionless phase speed $\alpha$ of the peaked solitary waves is unknown up to now. According to the linear UWM described in $\S$ 4.1, the peaked solitary waves exist only when

$$
\alpha^{2}=\frac{\tan k}{k}, \quad n \pi<k<n \pi+\frac{\pi}{2},
$$

where $n \geq 0$ is an integer. Note that here we consider only the primary decayparameter $0<k_{0}<\pi / 2$ with the definition $k=k_{0}$. If the above expression also holds for the fully nonlinear wave equations of the UWM, the auxiliary linear operator defined by (67) has the property

$$
\mathcal{L}\left\{\cos [k(z+1)] e^{-k x}\right\}=0, \quad x \geq 0, k=k_{0}>0,
$$

and the corresponding inverse operator of $\overline{\mathcal{L}}$ defined by (81) has the property

$$
\overline{\mathcal{L}}^{-1}\{\exp (-n k x)\}=\frac{\cos [n k(z+1)] \exp (-n k x)}{(n k)\left[\alpha^{2}(n k) \cos (n k)-\sin (n k)\right]}, \quad k>0, n \neq 1, x>0,
$$

where $n \geq 2$ is an integer. Note that the above expression does not hold when $n=1$. Fortunately, it is found that $R_{m}(x)$ indeed does not contain the term $\exp (-k x)$ as long as the phase speed is given by $\alpha^{2}=\tan (k) / k$, where $0<k<\pi / 2$. Mathematically, 
this is because the nonlinear terms of (61) do not contain the term $\exp (-k x)$ at all, since

$$
\exp (-m k x) \times \exp (-n k x)=e^{-(m+n) k x}
$$

with $m+n \geq 2$ for any integers $m \geq 1$ and $n \geq 1$. So do the linear terms of the nonlinear operator (61), since

$$
\begin{aligned}
& \left.\left\{\left(\alpha^{2} \frac{\partial^{2}}{\partial x^{2}}+\frac{\partial}{\partial z}\right) \sum_{n=1}^{+\infty} b_{n} \cos [n k(z+1)] \exp (-n k x)\right\}\right|_{z=0} \\
= & \sum_{n=1}^{+\infty}(n k)\left[\alpha^{2}(n k) \cos (n k)-\sin (n k)\right] b_{n} \exp (-n k x) \\
= & \sum_{n=2}^{+\infty}(n k)\left[\alpha^{2}(n k) \cos (n k)-\sin (n k)\right] b_{n} \exp (-n k x)
\end{aligned}
$$

does not contain the term $\exp (-k x)$, too. This is the essential reason why the phase speed

$$
\alpha=\sqrt{\frac{\tan (k)}{k}}
$$

given by the linear UWM still holds for the fully nonlinear wave equations (31) - (11) of the exact UWM! Thus, unlike the traditional smooth periodic and solitary progressive waves that are dispersive with wave height, the phase speed of the peaked solitary waves has nothing to do with the wave height: it is dispersive with the so-called "actual wavelength" $\lambda_{a}$ when wave height is fixed. More discussions about this point will be given in $\S 5$.

Keeping (88) in mind and using the property (87) of the inverse operator $\overline{\mathcal{L}}^{-1}$, it is straightforward to gain the common solution of the high-order deformation equation (80) to (82):

$$
\phi_{m}(x, z)=\phi_{m}^{*}(x, z)+A_{m} \cos [k(1+z)] e^{-k x}, \quad x>0,
$$

where $\phi_{m}^{*}(x, z)=\overline{\mathcal{L}}^{-1}\left[R_{m}(x)\right]$ is a special solution, and the coefficient $A_{m}$ is determined by the given wave height

$$
\sum_{n=1}^{m+1} \lim _{x \rightarrow 0} \zeta_{n}(x)=H_{w} .
$$

This is mainly because, according to (78),$\zeta_{m+1}(x)$ is dependent upon $\phi_{m}(x, z)$ that contains the unknown parameter $A_{m}$ for $m \geq 1$. Note that, according to (87), $\phi_{m}(x, z)$ is in the form of (57) and thus automatically satisfies the Laplace equation (3) in the domain $0<x<+\infty$, the bottom condition (6) and the bounded condition (12). Thus, using the explicit formulas given in the Appendix, it is computationally efficient to gain high-order analytic approximations successively, especially by means of the computer algebra system such as Mathematica and Maple, since our analytic approach needs only algebra computations. 
For example, using the initial guess (159) and (78), we directly have

$$
\begin{aligned}
\zeta_{1}(x) & =-\left.c_{\eta}\left(\alpha \frac{\partial \phi_{0}}{\partial x}-\frac{1}{2} \nabla \phi_{0} \cdot \nabla \phi_{0}\right)\right|_{z=0} \\
& =c_{\eta} A_{0} k\left[\alpha \cos (k) e^{-k x}+\frac{A_{0} k}{2} e^{-2 k x}\right], \quad x>0 .
\end{aligned}
$$

Thus, at the first-order of approximation, we have an algebraic equation for the given wave height

$$
H_{w}=c_{\eta} k A_{0}\left(\alpha \cos k+\frac{1}{2} k A_{0}\right),
$$

which gives two different solutions

$$
A_{0}=k^{-1}\left[-\alpha \cos k \pm \sqrt{\alpha^{2} \cos ^{2}(k)+2 H_{w} / c_{\eta}}\right] .
$$

We simply choice

$$
A_{0}=-k^{-1}\left[\alpha \cos k-\sqrt{\alpha^{2} \cos ^{2}(k)+2 H_{w} / c_{\eta}}\right]
$$

to calculate $A_{0}$ for a given $H_{w}$, since it has a smaller absolute value.

Furthermore, using the initial guess (59), we have

$$
\Delta_{0}^{\phi}=k A_{0}\left(\alpha^{2} k \cos k-\sin k\right) e^{-k x}+2 \alpha k^{3} A_{0}^{2} e^{-2 k x}+k^{4} A_{0}^{3} \cos (k) e^{-3 k x} .
$$

Using the phase speed (88), the term $\exp (-k x)$ of the above expression disappears, say,

$$
\Delta_{0}^{\phi}=2 \alpha k^{3} A_{0}^{2} e^{-2 k x}+k^{4} A_{0}^{3} \cos (k) e^{-3 k x}, \quad x>0 .
$$

Thus, the first-order deformation equation reads

$$
\nabla^{2} \phi_{1}(x, z)=0, \quad z \leq 0, \quad 0<x<+\infty
$$

subject to the boundary condition on the known free surface $z=0$ :

$$
\overline{\mathcal{L}}\left(\phi_{m}\right)=\left.\left(\alpha^{2} \frac{\partial^{2} \phi_{m}}{\partial x^{2}}+\frac{\partial \phi_{m}}{\partial z}\right)\right|_{z=0}=c_{\phi}\left[2 \alpha k^{3} A_{0}^{2} e^{-2 k x}+k^{4} A_{0}^{3} \cos (k) e^{-3 k x}\right],
$$

and the bottom condition

$$
\frac{\partial \phi_{1}}{\partial z}=0, \quad z=-1, \quad 0<x<+\infty .
$$

Using the property of the inverse operator (87), it is easy to gain the common solution

$$
\begin{aligned}
\phi_{1}(x, z) & =c_{\phi}\left\{\frac{\alpha k^{2} A_{0}^{2} \cos [2 k(z+1)] e^{-2 k x}}{2 \alpha^{2} k \cos (2 k)-\sin (2 k)}+\frac{k^{3} A_{0}^{3} \cos k \cos [3 k(z+1)] e^{-3 k x}}{3\left[3 \alpha^{2} k \cos (3 k)-\sin (3 k)\right]}\right\} \\
& +A_{1} \cos [k(z+1)] e^{-k x}, \quad 0<x<+\infty
\end{aligned}
$$


where $A_{1}$ is an unknown constant to be determined. Similarly, using (78), we gain $\zeta_{2}(x)$, which contains the unknown constant $A_{1}$. Then, for the given wave height $H_{w}$, we have a linear algebraic equation

$$
H_{w}=\lim _{x \rightarrow 0} \zeta_{1}(x)+\lim _{x \rightarrow 0} \zeta_{2}(x),
$$

which determines $A_{1}$. Then, $\phi_{1}(x, z)$ is completely determined. Similarly, we further gain $\phi_{2}(x, z), \zeta_{3}(x)$, and so on. Finally, using the symmetry (13), we gain the wave elevation $\zeta(x)$ and the velocities $u(x, z), v(x, z)$ in the interval $-\infty<x<0$ and $0<x<+\infty$. At $x=0$, the continuous horizontal velocity $u(0, z)=U(z)$ is given by (10), and the vertical velocity $v(0, z)=0$ is given directly by the symmetry condition (13). The convergence of the solution series is guaranteed by properly choosing the two convergence-control parameters $c_{\phi}$ and $c_{\eta}$ in the frame of the HAM. In this way, we gain the convergent series solutions of the peaked solitary waves in the whole domain $-\infty<x<+\infty$ by means of the UWM.

Our computations confirm that, for all $m \geq 0, R_{m}(x)$ in (81) indeed does not contain the term $\exp (-k x)$ at all. Thus, the fully nonlinear wave equations (3) $-(12)$ indeed give the same dimensionless phase speed $\alpha=\sqrt{\tan k / k}$ as that by the linear ones. Therefore, the phase speed of the peaked solitary wave indeed has nothing to do with the wave height $H_{w}$ : in fact, it is dependent upon the decay-parameter $k$, which determines the decay-length, i.e. the so-called "actual wavelength" $\lambda_{a}$, when wave height is fixed. In practice, the peaked solitary waves can be regarded to be dispersive (for a fixed wave height) with the "actual wavelength" that is a characteristic length in the horizontal direction. This is indeed completely different from the traditional periodic and solitary waves with smooth crest, which are dispersive with wave height that is a characteristic length in vertical direction. This unusual characteristic clearly demonstrates the novelty of the peaked solitary surface waves. We will discuss this interesting characteristic of the peaked solitary waves later.

Note that our HAM-based analytic approach mentioned above is rather similar to those by Liao \& Cheung [47] and Tao et al. [50] for the traditional smooth progressive waves in deep and finite water, except that we use here the symmetry condition (13), the evanescent-mode base-function (26), and besides regard the dimensionless phase speed $\alpha$ as a constant independent of wave height.

Finally, we should emphasize that, unlike perturbation methods, our HAM-based analytic approach does not need any assumptions about small/large physical parameters. More importantly, both of $\phi(x, z)$ and $\zeta(x)$ contain the two convergence-control parameters $c_{\phi}$ and $c_{\eta}$, which provide us a convenient way to guarantee the convergence of approximation series, as illustrated below.

\subsubsection{Convergence of series solution}

Note that, unlike perturbation results, $\phi_{m}(x, z)$ and $\zeta_{m}(x)$ gained in the abovementioned HAM-based approach contain the two convergence-control parameters $c_{\phi}$ 


\begin{tabular}{c|ccccc}
\hline \hline Order of approx. & $U(-1)$ & $U(-0.5)$ & $U(-0.25)$ & $U\left(H_{w}\right)$ & $\zeta^{\prime}\left(0_{+}\right)$ \\
\hline 1 & 0.07222 & 0.06570 & 0.05762 & 0.04289 & -0.04690 \\
3 & 0.06833 & 0.06236 & 0.05466 & 0.04205 & -0.04859 \\
5 & 0.06796 & 0.06219 & 0.05489 & $\mathbf{0 . 0 4 2 1 3}$ & $\mathbf{- 0 . 0 4 8 2 3}$ \\
10 & $\mathbf{0 . 0 6 7 9 9}$ & $\mathbf{0 . 0 6 2 2 1}$ & $\mathbf{0 . 0 5 4 9 0}$ & $\mathbf{0 . 0 4 2 1 3}$ & $\mathbf{- 0 . 0 4 8 2 3}$ \\
15 & $\mathbf{0 . 0 6 7 9 9}$ & $\mathbf{0 . 0 6 2 2 1}$ & $\mathbf{0 . 0 5 4 9 0}$ & $\mathbf{0 . 0 4 2 1 3}$ & $\mathbf{- 0 . 0 4 8 2 3}$ \\
20 & $\mathbf{0 . 0 6 7 9 9}$ & $\mathbf{0 . 0 6 2 2 1}$ & $\mathbf{0 . 0 5 4 9 0}$ & $\mathbf{0 . 0 4 2 1 3}$ & $\mathbf{- 0 . 0 4 8 2 3}$ \\
25 & $\mathbf{0 . 0 6 7 9 9}$ & $\mathbf{0 . 0 6 2 2 1}$ & $\mathbf{0 . 0 5 4 9 0}$ & $\mathbf{0 . 0 4 2 1 3}$ & $\mathbf{- 0 . 0 4 8 2 3}$ \\
\hline \hline
\end{tabular}

Table 1: Analytic approximations of $U(z)=u(0, z)$ and $\zeta^{\prime}\left(0_{+}\right)$in the case of $H_{w}=$ $1 / 20$ and $k=1$ by means of $c_{\phi}=-1$ and $c_{\eta}=-1$.

\begin{tabular}{c|ccccc}
\hline \hline Order of approx. & $U(-1)$ & $U(-0.5)$ & $U(-0.25)$ & $U\left(H_{w}\right)$ & $\zeta^{\prime}\left(0_{+}\right)$ \\
\hline 1 & -0.07047 & -0.06377 & -0.05101 & -0.03840 & 0.05248 \\
3 & -0.08133 & -0.06737 & -0.05218 & -0.03779 & 0.05220 \\
5 & -0.08140 & -0.06749 & -0.05218 & $\mathbf{- 0 . 0 3 7 7 2}$ & 0.05192 \\
10 & $\mathbf{- 0 . 0 8 1 4 5}$ & $\mathbf{- 0 . 0 6 7 5 0}$ & $\mathbf{- 0 . 0 5 2 1 8}$ & $\mathbf{- 0 . 0 3 7 7 2}$ & $\mathbf{0 . 0 5 1 8 3}$ \\
20 & $\mathbf{- 0 . 0 8 1 4 5}$ & $\mathbf{- 0 . 0 6 7 5 0}$ & $\mathbf{- 0 . 0 5 2 1 8}$ & $\mathbf{- 0 . 0 3 7 7 2}$ & $\mathbf{0 . 0 5 1 8 3}$ \\
25 & $\mathbf{- 0 . 0 8 1 4 5}$ & $\mathbf{- 0 . 0 6 7 5 0}$ & $\mathbf{- 0 . 0 5 2 1 8}$ & $\mathbf{- 0 . 0 3 7 7 2}$ & $\mathbf{0 . 0 5 1 8 3}$ \\
\hline \hline
\end{tabular}

Table 2: Analytic approximations of $U(z)=u(0, z)$ and $\zeta^{\prime}\left(0_{+}\right)$in the case of $H_{w}=$ $-1 / 20$ and $k=1$ by means of $c_{\phi}=-1$ and $c_{\eta}=-1$.

and $c_{\eta}$, which provide us a convenient way to guarantee the convergence of the series (76) and (77), as shown below.

First, let us consider the case of $k=1$ and $H_{w}=1 / 20$, with the corresponding dimensionless phase velocity $\alpha=\sqrt{\tan k / k} \approx 1.24796$. Since the wave height is only $5 \%$ of the water depth $D$, the nonlinearity is weak. Thus, following Liao \& Cheung [47] and Tao et al. [50], we choose $c_{\phi}=-1$ and $c_{\eta}=-1$ for such a kind of weakly nonlinear wave problem. It is found that, the corresponding series of analytic approximations indeed converge quickly, as shown in Table 1 for $\zeta^{\prime}\left(0_{+}\right)$and the horizontal velocity $U(z)=u(0, z)$ at $x=0$ when $z=-1, z=-1 / 2, z=-1 / 4$ and $z=H_{w}$, respectively, where $0_{+}$denotes $x \rightarrow 0$ from the right along the $x$ axis. It is found that the velocity potential $\phi(x, z)$ converges quickly in the domain $x \in(0,+\infty)$ and $z \leq \zeta(x)$, as shown in Fig. [ for the corresponding horizontal velocity profile $U(z)=u(0, z)$ at crest $(x=0)$. This confirms that the peaked solitary wave is indeed a solution of the UWM based on the symmetry and the fully nonlinear wave equations (3i) - (12).

Secondly, let us consider the case with $k=1$ and $H_{w}=-1 / 20$, with the same dimensionless phase velocity $\alpha=c / \sqrt{g D} \approx 1.24796$. It is found that the correspond- 


\begin{tabular}{c|ccccc}
\hline \hline Order of approx. & $U(-1)$ & $U(-0.5)$ & $U(-0.25)$ & $U\left(H_{w}\right)$ & $\zeta^{\prime}\left(0_{+}\right)$ \\
\hline 1 & 0.1696 & 0.1543 & 0.1347 & 0.09362 & -0.08561 \\
3 & 0.1246 & 0.1180 & 0.1090 & 0.08837 & -0.09332 \\
5 & 0.1270 & 0.1196 & 0.1098 & 0.08813 & -0.09142 \\
10 & 0.1254 & 0.1183 & 0.1090 & 0.08788 & -0.09285 \\
15 & $\mathbf{0 . 1 2 5 4}$ & $\mathbf{0 . 1 1 8 3}$ & $\mathbf{0 . 1 0 9 0}$ & $\mathbf{0 . 0 8 7 8 9}$ & $\mathbf{- 0 . 0 9 2 9 9}$ \\
20 & $\mathbf{0 . 1 2 5 4}$ & $\mathbf{0 . 1 1 8 3}$ & $\mathbf{0 . 1 0 9 0}$ & $\mathbf{0 . 0 8 7 8 9}$ & $\mathbf{- 0 . 0 9 2 9 9}$ \\
25 & $\mathbf{0 . 1 2 5 4}$ & $\mathbf{0 . 1 1 8 3}$ & $\mathbf{0 . 1 0 9 0}$ & $\mathbf{0 . 0 8 7 8 9}$ & $\mathbf{- 0 . 0 9 2 9 9}$ \\
\hline \hline
\end{tabular}

Table 3: Analytic approximations of $U(z)=u(0, z)$ and $\zeta^{\prime}\left(0_{+}\right)$in the case of $H_{w}=$ $1 / 10$ and $k=1$ by means of $c_{\phi}=-1 / 2$ and $c_{\eta}=-1$.

\begin{tabular}{c|ccccc}
\hline \hline Order of approx. & $U(-1)$ & $U(-0.5)$ & $U(-0.25)$ & $U\left(H_{w}\right)$ & $\zeta^{\prime}\left(0_{+}\right)$ \\
\hline 1 & -0.1418 & -0.1191 & -0.09328 & -0.07474 & 0.1091 \\
3 & -0.1704 & -0.1343 & -0.09684 & -0.07211 & 0.1130 \\
5 & -0.1768 & -0.1368 & -0.09664 & -0.07070 & 0.1107 \\
10 & -0.1801 & -0.1379 & -0.09650 & -0.07016 & 0.1079 \\
15 & -0.1805 & -0.1380 & -0.09649 & -0.07013 & 0.1075 \\
20 & $\mathbf{- 0 . 1 8 0 6}$ & $\mathbf{- 0 . 1 3 8 0}$ & $\mathbf{- 0 . 0 9 6 4 8}$ & $\mathbf{- 0 . 0 7 0 1 2}$ & $\mathbf{0 . 1 0 7 5}$ \\
25 & $\mathbf{- 0 . 1 8 0 6}$ & $\mathbf{- 0 . 1 3 8 0}$ & $\mathbf{- 0 . 0 9 6 4 8}$ & $\mathbf{- 0 . 0 7 0 1 2}$ & $\mathbf{0 . 1 0 7 5}$ \\
\hline \hline
\end{tabular}

Table 4: Analytic approximations of $U(z)=u(0, z)$ and $\zeta^{\prime}\left(0_{+}\right)$in the case of $H_{w}=$ $-1 / 10$ and $k=1$ by means of $c_{\phi}=-3 / 4$ and $c_{\eta}=-1$.

ing series of analytic approximations given by $c_{\phi}=-1$ and $c_{\eta}=-1$ converge quickly in the domain $x \geq 0$, as shown in Table 2 for $\zeta^{\prime}\left(0_{+}\right)$and the horizontal velocity $U(z)=u(0, z)$ at crest $(x=0)$ when $z=-1,-0.5,-0.25$ and $z=H_{w}$, respectively. Besides, the corresponding velocity potential $\phi(x, z)$ converges quickly in the domain $x \in(0,+\infty)$ and $z \leq \zeta(x)$, as shown in Fig. 5 for the horizontal velocity profile $U(z)=u(0, z)$ at crest. This confirms that the peaked solitary wave in the form of depression is also a solution of the UWM, too.

Similarly, in the case of $k=1 / 2$ and $H_{w}= \pm 1 / 20$, with the corresponding dimensionless phase speed $\alpha \approx 1.04528$, we gain convergent series solutions of $\phi(x, z)$ and $\zeta(x)$ in the domain $x \in(0,+\infty)$ and $z \leq \zeta(x)$ by means of $c_{\phi}=-1$ and $c_{\eta}=-1$, respectively.

Note that, using the symmetry conditions (13) and (14), we can expand all of these convergent series solutions into the whole domain $-\infty<x<+\infty$. Thus, the UWM indeed admits the peaked solitary waves! In other words, the UWM unifies both of the smooth and peaked waves, for the first time, to the best of the author's 


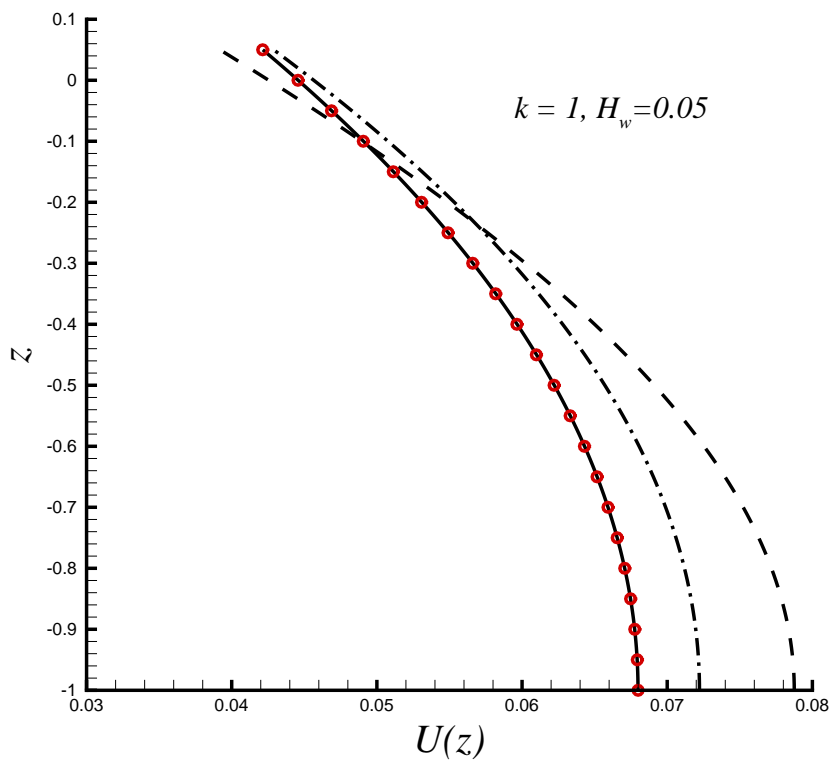

Figure 4: Analytic approximations of the dimensionless horizontal velocity profile $U(z)=u(0, z)$ beneath the crest in the case of $k=1$ and $H_{w}=0.05$ given by $c_{\phi}=-1$ and $c_{\eta}=-1$. Dashed-line: zeroth-order of approx.; Dash-dotted line: 1st-order of approx.; Solid line: 4th-order of approx.; Symbols: 25th-order of approximation.

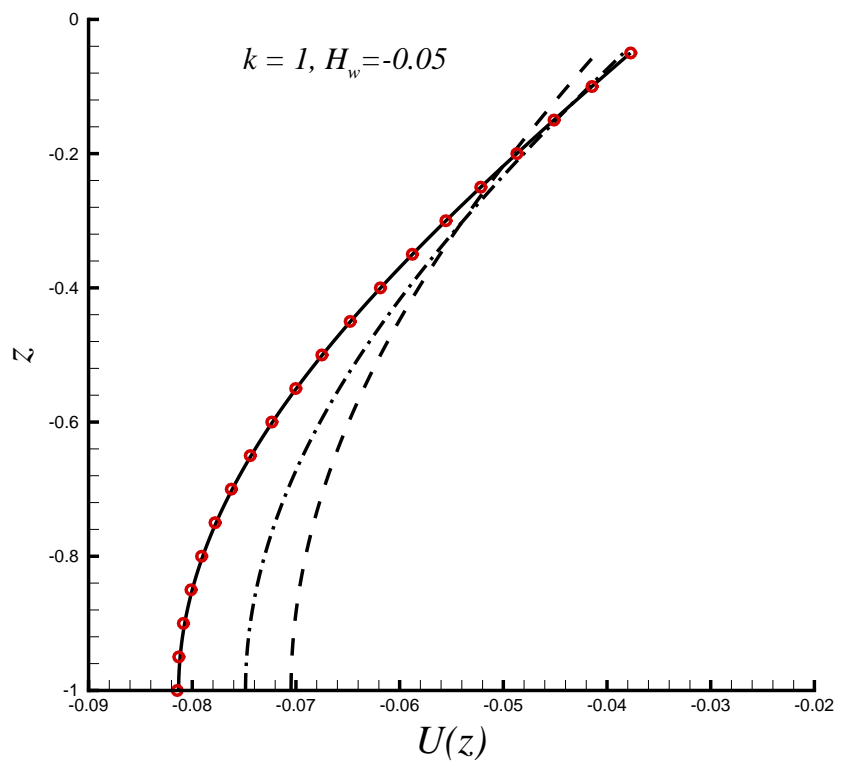

Figure 5: Analytic approximations of the dimensionless horizontal velocity profile $U(z)=u(0, z)$ beneath the crest in the case of $k=1$ and $H_{w}=-0.05$ given by $c_{\phi}=$ -1 and $c_{\eta}=-1$. Dashed-line: zeroth-order of approx.; Dash-dotted line: 1st-order of approx.; Solid line: 4th-order of approx.; Symbols: 25th-order of approximation. 


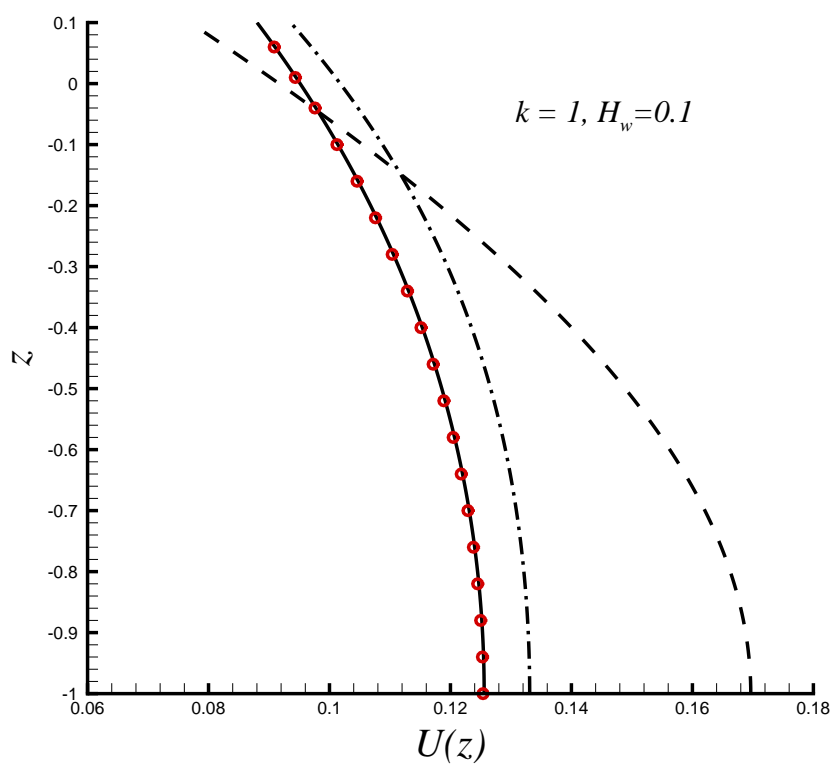

Figure 6: Analytic approximations of the dimensionless horizontal velocity profile $U(z)=u(0, z)$ beneath the crest in the case of $k=1$ and $H_{w}=0.1$ given by $c_{\phi}=-1 / 2$ and $c_{\eta}=-1$. Dashed-line: zeroth-order of approx.; Dash-dotted line: 2nd-order of approx.; Solid line: 6th-order of approx.; Symbols: 25th-order of approximation.

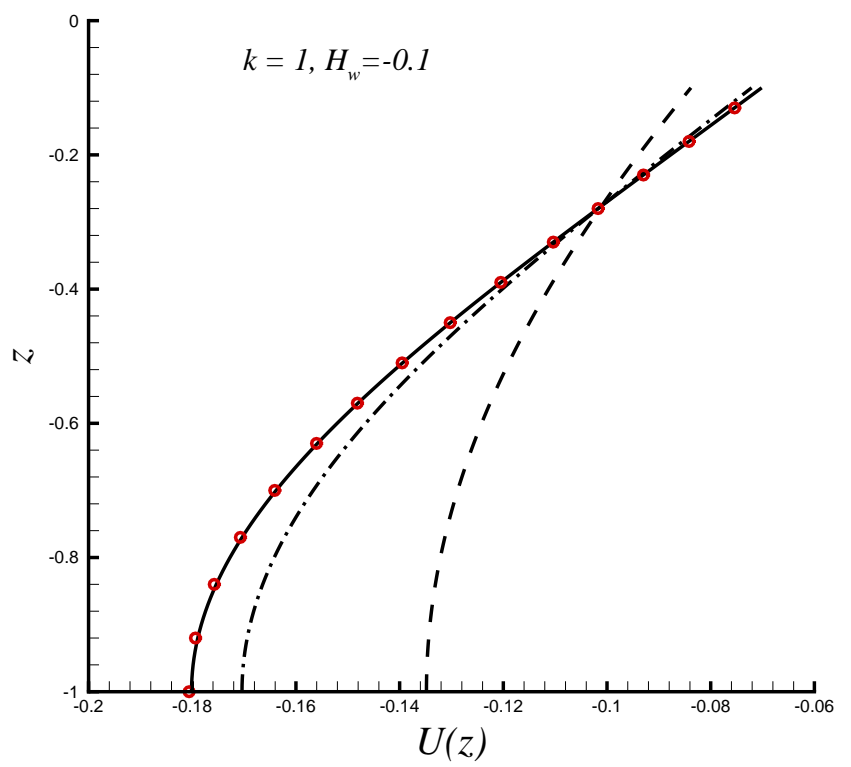

Figure 7: Analytic approximations of the dimensionless horizontal velocity profile $U(z)=u(0, z)$ beneath the crest in the case of $k=1$ and $H_{w}=-0.1$ given by $c_{\phi}=$ $-3 / 4$ and $c_{\eta}=-1$. Dashed-line: zeroth-order of approx.; Dash-dotted line: 1st-order of approx.; Solid line: 10th-order of approx.; Symbols: 25th-order of approximation. 


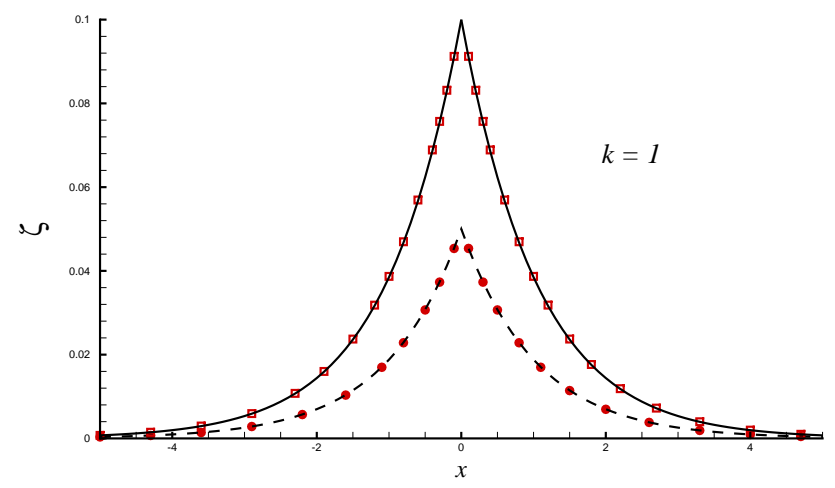

Figure 8: Analytic approximations of elevation of the peaked solitary waves when $k=1$ (corresponding to $c / \sqrt{g D}=1.24796$ ). Solid line: 5th-order approximation when $H_{w}=0.1$ given by $c_{\phi}=-0.5$ and $c_{\eta}=-1$; Filled circles: 25th-order approximation when $H_{w}=0.1$ given by $c_{\phi}=-0.5$ and $c_{\eta}=-1$; Dashed line: 5th-order approximation when $H_{w}=0.05$ given by $c_{\phi}=-1$ and $c_{\eta}=-1$; Open circles: 25thorder approximation when $H_{w}=0.05$ given by $c_{\phi}=-1$ and $c_{\eta}=-1$.

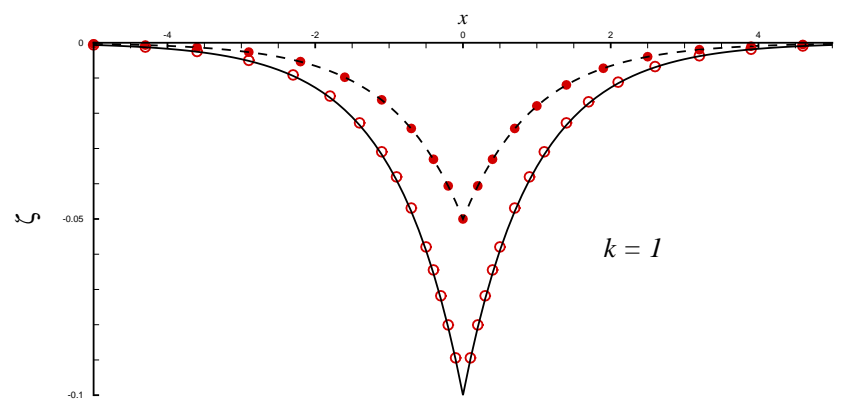

Figure 9: Analytic approximations of elevation of the peaked solitary waves when $k=1$ (corresponding to $c / \sqrt{g D}=1.24796$ ). Solid line: 5th-order approximation when $H_{w}=-0.1$ given by $c_{\phi}=-0.75$ and $c_{\eta}=-1$; Filled circles: 25th-order approximation when $H_{w}=-0.1$ given by $c_{\phi}=-0.75$ and $c_{\eta}=-1$; Dashed line: 5th-order approximation when $H_{w}=-0.05$ given by $c_{\phi}=-1$ and $c_{\eta}=-1$; Open circles: 25th-order approximation when $H_{w}=-0.05$ given by $c_{\phi}=-1$ and $c_{\eta}=-1$. 


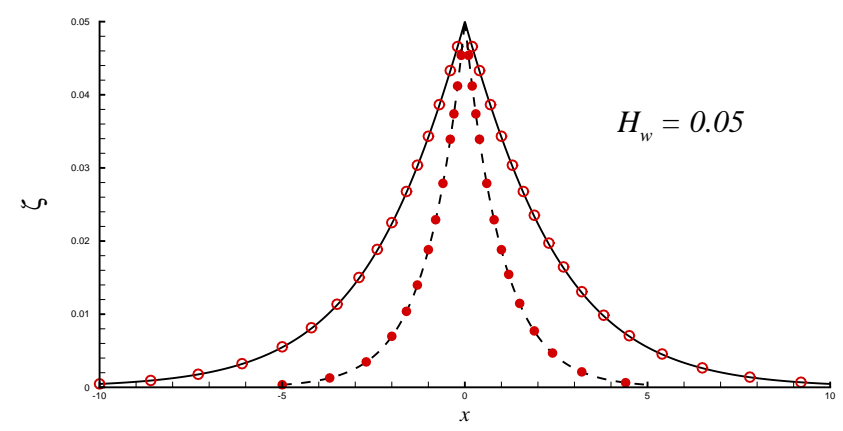

Figure 10: Analytic approximations of $\zeta(x)$ of the peaked solitary waves when $H_{w}=$ 0.05 by means of $c_{\phi}=-1$ and $c_{\eta}=-1$. Solid line: 5th-order approximation when $k=$ $1 / 2$ (corresponding to $c / \sqrt{g D}=1.04528$ ); Filled circles: 25th-order approximation when $k=1 / 2$; Dashed line: 5th-order approximation when $k=1$ (corresponding to $c / \sqrt{g D}=1.24796)$; Open circles: 25th-order approximation when $k=1$.

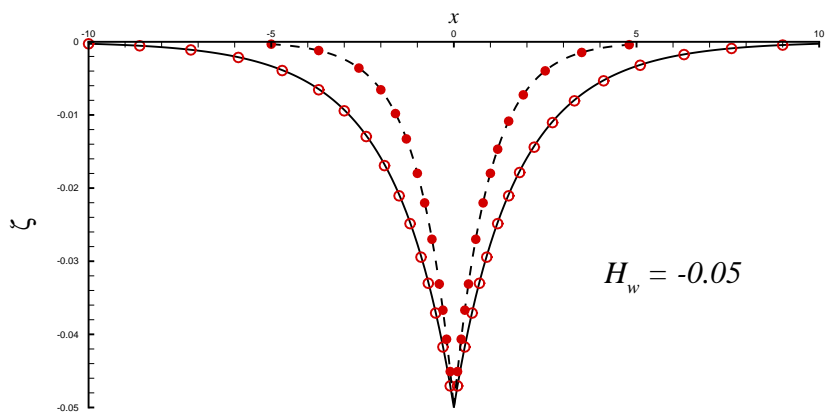

Figure 11: Analytic approximations of $\zeta(x)$ of the peaked solitary waves when $H_{w}=-0.05$ by means of $c_{\phi}=-1$ and $c_{\eta}=-1$. Solid line: 5th-order approximation when $k=1 / 2$ (corresponding to $c / \sqrt{g D}=1.04528$ ); Filled circles: 25th-order approximation when $k=1 / 2$; Dashed line: 5th-order approximation when $k=1$ (corresponding to $c / \sqrt{g D}=1.24796$; Open circles: 25th-order approximation when $k=1$. 
knowledge. Mathematically, it reveals that the peaked solitary waves are consistent with the traditional smooth waves and thus are as acceptable as them in the frame of inviscid fluid.

Furthermore, let us consider the case of $k=1$ and $H_{w}=0.1$, with the corresponding dimensionless phase velocity $\alpha \approx 1.24796$. Since the wave weight increases to $10 \%$ of water depth, the nonlinearity becomes stronger. As suggested by Liao \& Cheung [47] and Tao et al. [50], we should choose the convergence-control parameters $c_{\phi}$ and $c_{\eta}$ with smaller absolute values for high nonlinearity. It is found that the series of the analytic approximations given by $c_{\phi}=-1 / 2$ and $c_{\eta}=-1$ converges quickly, as shown in Table 3 and Fig. 6 for the horizontal velocity profile $U(z)=u(0, z)$ at crest. Similarly, in the case of $k=1$ and $H_{w}=-0.1$, we gain convergent series solution by means of $c_{\phi}=-3 / 4$ and $c_{\eta}=-1$, as shown in Table 4 and Fig. 7 . This illustrates that the two convergence-control parameters $c_{\phi}$ and $c_{\eta}$ indeed provide us a convenient way to guarantee the convergence of approximation series. Note that the absolute value of the horizontal velocity on bottom in the case of $H_{w}=-0.1$ is $44 \%$ larger than that in the case of $H_{w}=0.1$. So, due to the nonlinearity, there does not exist a symmetry between these two wave elevations for $H_{w}=+0.1$ and $H_{w}=-0.1$, respectively.

It should be emphasized that, in the case of $k=1$, we gain convergent series solutions of the peaked solitary waves with the same phase speed but different positive and negative values of $H_{w}$, such as $H_{w}= \pm 0.05$ and $H_{w}= \pm 0.1$, respectively. This confirms that the phase speed of the peaked solitary waves indeed has nothing to do with the wave height. As mentioned in $\S 4.1$, for a given wave height, the value of $k$ determines the decay length, which is the characteristic length of the peaked solitary waves in the horizontal direction, corresponding to the so-called "actual wavelength" $\lambda_{a}$ defined by (46). Thus, unlike the traditional smooth waves that are dispersive with wave height, the peaked solitary waves are dispersive (for a fixed wave height) with the "actual wavelength", although their phase speed has nothing to do with the wave height. This is an unusual characteristic of the peaked solitary waves.

Finally, using the symmetry (13), it is straightforward to gain the wave elevation in the whole interval $-\infty<x<+\infty$. As shown in Figs. 8 to 11, the wave elevations $\zeta(x)$ also converge quickly in all of above-mentioned cases. In case of $k=1$, the wave elevations for $H_{w}= \pm 0.1$ are compared with those for $H_{w}= \pm 0.05$, as shown in Figs. 8 and 9. It is found that, for the same value of $k$, the larger the value of $\left|H_{w}\right|$, the faster $\zeta(x)$ decays to zero. Note also that the wave elevation $\zeta(x)$ with larger $k$ decays to 0 more quickly, as shown in Figs. 10 and 11. In other words, the larger the value of $k$, the faster $\zeta(x) \rightarrow 0$. This provides us a physical meaning of the parameter $k$. For this reason, we call $k$ the decay-parameter, which determines the so-called decay-length $\lambda_{a}$, defined by (46).

Note that, according to the convergence theorem generally proved by Liao [16, 19] in the frame of the HAM, each series solution of the peaked solitary waves given by the HAM satisfies its original equations, as long as it is convergent. So, all of these convergent series of $\phi(x, z)$ and $\zeta(x)$ are solutions of the unified wave model (UWM) based on the symmetry and the fully nonlinear wave equations (3) - (12), as further 
confirmed below.

\subsubsection{Validation check of analytic approximations}

Note that the velocity potential $\phi(x, z)$ is expressed in the form (57), which automatically satisfies the Laplace equation (31) in the interval $0<x<+\infty$, the bottom condition (6), and the bounded condition (12). Thus, it is only necessary for us to check the two nonlinear boundary conditions (44) and (5) defined on the unknown wave elevation $\zeta(x)$.

To check the validation of our analytic approximations, we define the averaged residual squares of the two free surface boundary conditions

$$
\begin{aligned}
& \mathcal{E}_{m}^{\phi}\left(c_{\phi}, c_{\eta}\right)=\left.\frac{1}{M} \sum_{n=1}^{M}(\mathcal{N}[\check{\phi}(x, z)])^{2}\right|_{x=x_{n}, z=\zeta\left(x_{n}\right)}, \\
& \mathcal{E}_{m}^{\zeta}\left(c_{\phi}, c_{\eta}\right)=\left.\frac{1}{M} \sum_{n=1}^{M}\left[\check{\zeta}(x)-\alpha \frac{\partial \check{\phi}}{\partial x}+\frac{1}{2} \nabla \check{\phi} \cdot \nabla \check{\phi}\right]^{2}\right|_{x=x_{n}, z=\zeta\left(x_{n}\right)},
\end{aligned}
$$

where

$$
\check{\phi}(x, z)=\sum_{n=0}^{m} \phi_{n}(x, z), \quad \check{\zeta}=\sum_{n=1}^{m} \zeta_{n}(x)
$$

are the $m$ th-order approximation of $\phi(x, z)$ and $\zeta(x)$, respectively, and

$$
x_{n}=n\left(\frac{x_{R}}{M}\right), \quad 1 \leq n \leq M,
$$

with large enough $x_{R}$ and $M$. For all results given below, we choose $x_{R}=10$ and $M=100$, if not mentioned. Since the potential velocity $\phi(x, z)$ and the wave elevation $\zeta(x)$ decay exponentially in the horizontal direction, $x_{R}=10$ is large enough.

In case of $k=1$ and $H_{w}= \pm 0.05$, the averaged residual squares $\mathcal{E}_{m}^{\phi}$ and $\mathcal{E}_{m}^{\zeta}$ of the corresponding analytic approximations obtained by $c_{\phi}=-1$ and $c_{\eta}=-1$ decay quickly to the level $10^{-25}$ as the order of approximation increases to 25 , as shown in Table 5. In other words, our 25th-order approximation of $\phi(x, z)$ and $\zeta(x)$ satisfies the Laplace equation (3) in the interval $0<x<+\infty$, the bottom condition (6) and the bounded condition (12) exactly, and besides the two nonlinear free surface boundary conditions (44) and (5) very accurately (to the level $10^{-25}$ ). In addition, $u(0, z)$ determined by (10) converges quickly so that $U(z)$ is uniquely determined. Therefore, our convergent analytic approximation is a very accurate solution of the UWM based on the symmetry and the fully nonlinear wave equations (31) - (12). Similarly, $\mathcal{E}_{m}^{\phi}$ and $\mathcal{E}_{m}^{\zeta}$ decays to the level $10^{-13}$ in the case of $k=1$ and $H_{w}= \pm 0.1$, and to the level $10^{-18}$ in the case of $k=1 / 2$ and $H_{w}= \pm 0.05$, respectively, as shown in Tables 6 and 7 . Theses guarantee that the corresponding analytic approximations of $\phi(x, z)$ and $\zeta(x)$ are indeed quite accurate solutions of the UWM, respectively. All of these confirm once again the convergence theorem generally proved by Liao [16, 19] 


\begin{tabular}{c|cc|cc}
\hline \hline Order of approx. & \multicolumn{2}{|c|}{$H_{w}=0.05$} & \multicolumn{2}{c}{$H_{w}=-0.05$} \\
$m$ & $\mathcal{E}_{m}^{\phi}$ & $\mathcal{E}_{m}^{\zeta}$ & $\mathcal{E}_{m}^{\phi}$ & $\mathcal{E}_{m}^{\zeta}$ \\
\hline 1 & $6.59 \times 10^{-7}$ & $9.21 \times 10^{-9}$ & $2.25 \times 10^{-6}$ & $9.39 \times 10^{-9}$ \\
3 & $1.40 \times 10^{-8}$ & $1.57 \times 10^{-9}$ & $8.09 \times 10^{-9}$ & $5.90 \times 10^{-10}$ \\
5 & $3.32 \times 10^{-11}$ & $2.90 \times 10^{-12}$ & $7.80 \times 10^{-11}$ & $1.53 \times 10^{-11}$ \\
10 & $9.71 \times 10^{-15}$ & $7.00 \times 10^{-16}$ & $8.96 \times 10^{-16}$ & $2.42 \times 10^{-16}$ \\
15 & $7.33 \times 10^{-19}$ & $1.68 \times 10^{-19}$ & $6.97 \times 10^{-20}$ & $1.05 \times 10^{-20}$ \\
20 & $4.43 \times 10^{-22}$ & $4.83 \times 10^{-23}$ & $2.40 \times 10^{-24}$ & $6.45 \times 10^{-25}$ \\
25 & $2.23 \times 10^{-25}$ & $2.09 \times 10^{-27}$ & $4.56 \times 10^{-28}$ & $3.96 \times 10^{-29}$ \\
\hline \hline
\end{tabular}

Table 5: Averaged residual squares of the two nonlinear free boundary conditions (4) and (5i) in the case of $k=1$ and $H_{w}= \pm 0.05$ by means of $c_{\phi}=-1$ and $c_{\eta}=-1$, with the corresponding dimensionless phase speed $c / \sqrt{g D}=1.24796$.

\begin{tabular}{c|cc|cc}
\hline \hline $\begin{array}{c}\text { Order of approx. } \\
m\end{array}$ & \multicolumn{2}{|c|}{$H_{w}=0.1$} & \multicolumn{2}{c}{$H_{w}=-0.1$} \\
& \multicolumn{2}{c|}{$\left(c_{\phi}=-0.5, c_{\eta}=-1\right)$} & \multicolumn{2}{c}{$\left(c_{\phi}=-0.75, c_{\eta}=-1\right)$} \\
$\mathcal{E}_{m}^{\phi}$ & $\mathcal{E}_{m}^{\zeta}$ & $\mathcal{E}_{m}^{\phi}$ & $\mathcal{E}_{m}^{\zeta}$ \\
\hline 1 & $1.48 \times 10^{-4}$ & $7.21 \times 10^{-7}$ & $5.89 \times 10^{-5}$ & $3.67 \times 10^{-7}$ \\
3 & $1.63 \times 10^{-7}$ & $8.63 \times 10^{-8}$ & $5.84 \times 10^{-7}$ & $1.21 \times 10^{-7}$ \\
5 & $1.39 \times 10^{-7}$ & $3.96 \times 10^{-10}$ & $6.83 \times 10^{-7}$ & $1.11 \times 10^{-8}$ \\
10 & $5.96 \times 10^{-10}$ & $3.09 \times 10^{-11}$ & $8.63 \times 10^{-9}$ & $2.31 \times 10^{-10}$ \\
15 & $1.30 \times 10^{-12}$ & $3.70 \times 10^{-14}$ & $3.29 \times 10^{-11}$ & $1.95 \times 10^{-12}$ \\
20 & $4.34 \times 10^{-13}$ & $1.88 \times 10^{-15}$ & $1.17 \times 10^{-12}$ & $3.69 \times 10^{-14}$ \\
25 & $2.25 \times 10^{-13}$ & $4.52 \times 10^{-16}$ & $2.51 \times 10^{-14}$ & $8.11 \times 10^{-16}$ \\
\hline \hline
\end{tabular}

Table 6: Averaged residual squares of the two nonlinear free boundary conditions (4) and (5) in the case of $k=1$ and $H_{w}= \pm 0.1$, with the corresponding dimensionless phase speed $c / \sqrt{g D}=1.24796$. 


\begin{tabular}{c|cc|cc}
\hline \hline Order of approx. & \multicolumn{2}{|c|}{$H_{w}=0.05$} & \multicolumn{2}{c}{$H_{w}=-0.05$} \\
$m$ & $\mathcal{E}_{m}^{\phi}$ & $\mathcal{E}_{m}^{\zeta}$ & $\mathcal{E}_{m}^{\phi}$ & $\mathcal{E}_{m}^{\zeta}$ \\
\hline 1 & $2.75 \times 10^{-8}$ & $1.04 \times 10^{-6}$ & $3.74 \times 10^{-7}$ & $9.09 \times 10^{-7}$ \\
3 & $3.76 \times 10^{-10}$ & $4.85 \times 10^{-8}$ & $4.48 \times 10^{-10}$ & $9.61 \times 10^{-9}$ \\
5 & $4.13 \times 10^{-12}$ & $2.00 \times 10^{-9}$ & $4.85 \times 10^{-13}$ & $4.18 \times 10^{-12}$ \\
10 & $1.80 \times 10^{-14}$ & $2.21 \times 10^{-12}$ & $1.08 \times 10^{-16}$ & $3.16 \times 10^{-17}$ \\
15 & $5.23 \times 10^{-15}$ & $1.55 \times 10^{-15}$ & $7.93 \times 10^{-20}$ & $1.06 \times 10^{-19}$ \\
20 & $1.86 \times 10^{-16}$ & $1.50 \times 10^{-16}$ & $1.67 \times 10^{-23}$ & $1.52 \times 10^{-24}$ \\
25 & $2.56 \times 10^{-18}$ & $9.46 \times 10^{-18}$ & $3.87 \times 10^{-29}$ & $2.57 \times 10^{-28}$ \\
\hline \hline
\end{tabular}

Table 7: Averaged residual squares of the two nonlinear free boundary conditions (4) and (5) in the case of $k=1 / 2$ and $H_{w}= \pm 0.05$ by means of $c_{\phi}=-1$ and $c_{\eta}=-1$, with the corresponding dimensionless phase speed $c / \sqrt{g D}=1.04528$.

in the frame of the HAM: each convergent series solution given by the HAM satisfies its original equations.

In fact, one can choose the optimal values of $c_{\phi}$ and $c_{\eta}$ by the minimum of $\mathcal{E}_{m}^{\phi}\left(c_{\phi}, c_{\eta}\right)$ and $\mathcal{E}_{m}^{\zeta}\left(c_{\phi}, c_{\eta}\right)$, say,

$$
\frac{\partial \mathcal{E}_{m}^{\phi}\left(c_{\phi}, c_{\eta}\right)}{\partial c_{\phi}}=0, \quad \frac{\partial \mathcal{E}_{m}^{\zeta}\left(c_{\phi}, c_{\eta}\right)}{\partial c_{\zeta}}=0 .
$$

It is found that, by means of the optimal values of $c_{\phi}$ and $c_{\eta}$, the corresponding series of analytic approximations often converge more quickly.

All of these demonstrate that the convergent series of the peaked solitary waves obtained by our HAM-based approach are indeed the solutions of the unified wave model (UWM) based on the symmetry and the fully nonlinear wave equations (3) (12).

\subsection{Characteristics of peaked solitary surface waves}

Based on the so-called evanescent base-functions (26), the peaked solitary waves governed by the exact UWM have some unusual characteristics that are quite different from those of the traditional smooth waves.

First, the peaked solitary waves have a peaked wave crest, since $\zeta^{\prime}(x)$ is discontinuous at $x=0$, i.e. $\zeta^{\prime}\left(0_{+}\right)=-\zeta^{\prime}\left(0_{-}\right) \neq 0$, where $0_{+}$and $0_{-}$denote $x \rightarrow 0$ from the right and left along the $x$ axis, respectively. For example, in the case of $H_{w}=+0.1$ and $k=1$, we have $\zeta^{\prime}\left(0_{+}\right)=-0.09299$, but $\zeta^{\prime}\left(0_{-}\right)=0.09299$, respectively. This is quite different from traditional smooth periodic and solitary waves which are infinitely differentiable everywhere. 
Secondly, the peaked solitary waves may be in the form of depression, which has been reported for internal waves but never for surface ones, to the best of the author's knowledge. Mathematically, it is straightforward to gain such kind of solitary waves in a depression form even by means of the linear UWM, as shown in $\S 4.1$.

Third, unlike traditional smooth periodic and solitary waves which are dispersive with wave height, the dimensionless phase speed of the peaked solitary waves has nothing to do with the wave height, but depends only upon the so-called decay-parameter $k$. For a fixed wave height, the decay-parameter $k$ determines the decay-length $\lambda_{a}$, which is a characteristic length of the peaked solitary waves in the horizontal direction, called the "actual wavelength". Thus, unlike the traditional smooth waves, the peaked solitary waves are dispersive (for a fixed wave height) with "actual wavelength" $\lambda_{a}$, defined by (46). So, in the same water depth $D$, the peaked solitary waves with the same $k$ but different wave height $H_{w}$ may propagate with the same phase speed, where $H_{w}$ may be either positive or negative. For example, it is found that, in the case of $k=1$, all of the peaked solitary waves with $H_{w}= \pm 0.1$ or $H_{w}= \pm 0.5$ propagate with the same phase speed $c \approx 1.24796 \sqrt{g D}$ : in these cases, we gain different series solutions with the same phase speed, as shown in $\S 4.2 .2$. On the other side, the peaked solitary waves with the same wave height $H_{w}$ but different decay-parameter $k$ (corresponding to different "actual wavelengths") may propagate with different phase speed! These are completely different from the traditional periodic and solitary waves with smooth crest. In summary, unlike the traditional smooth periodic and solitary waves which are dispersive with wave height that is a characteristic length in the vertical direction, the phase speed of peaked solitary waves has nothing to do with wave height: in fact, they are dispersive (for a fixed wave height) with "actual wavelength", a characteristic length in the horizontal direction.

Furthermore, as shown in Tables 1 to 4 and Figs. 2 to 7 , the horizontal bottom velocity of the peaked solitary waves is always larger than that on free surface. For example, in the case of $k=1$ and $H_{w}=0.1$, the bottom horizontal velocity at crest is $43 \%$ larger than that on free surface, as shown in Table 3. Especially, as shown in Table 4, in the case of $k=1$ and $H_{w}=-0.1$, the horizontal bottom velocity at crest is even $158 \%$ larger than that on free surface! In general, for the same $x$, the horizontal bottom velocity $u(x,-1)$ has always a larger absolute value than $u(x, \zeta)$ on the free surface, as shown in Figs. 12 and 13. This is quite different from the traditional smooth periodic and solitary waves, whose horizontal bottom velocity always decays exponentially from free surface to bottom. The similar characteristic was reported for the peaked solitary waves given by the linear UWM in $\S 4.1$.

In addition, as mentioned in $\S 4.1$, the kinetic energy of peaked solitary waves given by the linear UWM is independent of $z$. Using the exact UWM, we obtain the similar conclusions. For example, in case of $k=1$, it is found that the kinetic energy of peaked solitary waves on bottom may be $13.1 \%$ larger than that on free surface when $H_{w}=-1 / 20$, and is at most only $10.4 \%$ smaller when $H_{w}=1 / 20$. Therefore, from free surface to bottom, the kinetic energy of peaked solitary waves either increases or decays a little. This is quite different from the traditional smooth waves whose kinetic energy decreases exponentially from free surface to bottom. 


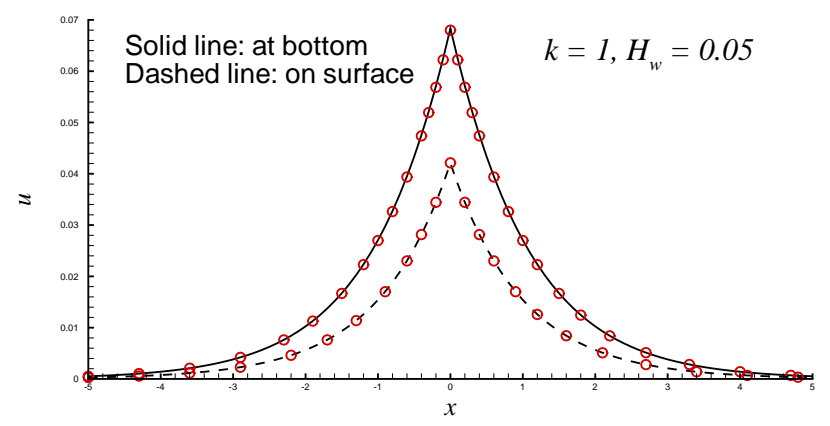

Figure 12: Horizontal velocity at bottom and on free surface when $k=1$ and $H_{w}=0.05$ by means of $c_{\phi}=-1$ and $c_{\eta}=-1$. Solid line: 3rd-order approx. of $u(x,-1)$ (at bottom); Dashed line: 3rd-order approx. of $u(x, \zeta(x))$ (on free surface); Symbols: the corresponding 25th-order approximations.

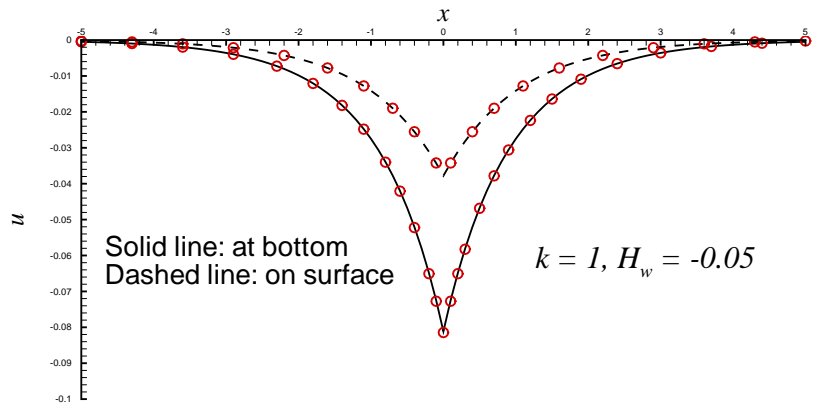

Figure 13: Horizontal velocity at bottom and on free surface when $k=1$ and $H_{w}=-0.05$ by means of $c_{\phi}=-1$ and $c_{\eta}=-1$. Solid line: 3rd-order approx. of $u(x,-1)$ (at bottom); Dashed line: 3rd-order approx. of $u(x, \zeta(x))$ (on free surface); Symbols: the corresponding 25th-order approximations. 
All of these unusual characteristics clearly indicate the novelty of the peaked solitary waves. Note that the peaked solitary waves given by the linear UWM in $\S 4.1$ have the rather similar characteristics as mentioned above.

\subsection{Kelvin's theorem for peaked solitary waves}

As mentioned above, at crest, the peaked solitary waves given by the unified wave model (UWM) have a discontinuous vertical velocity and the discontinuous 1st-derivative of wave elevation. Does Kelvin's theorem still hold for them?

Without loss of generality, let us consider the interface of two fluids in general. Let $\mathbf{u}_{i}$ and $\rho_{i}$ denote the velocity vector and density of the two fluids, where $i=1,2$. Taking a closed curve $\mathcal{C}$ moving with the fluid, we have the circulation

$$
\Gamma=\oint_{\mathcal{C}} \mathbf{u} \cdot \mathbf{d r} .
$$

Obviously, it holds the Kelvin's theorem $d \Gamma / d t=0$ in each fluid. Then, consider a closed curve $\mathcal{C}$ cross the interface of the two fluids. Let $Q_{1}$ and $Q_{2}$ denote the two points of intersection of the curve $\mathcal{C}$ and the interface. The circulation reads

$$
\Gamma=\int_{\mathcal{C}_{1}} \mathbf{u}_{1} \cdot \mathbf{d} \mathbf{r}+\int_{\mathcal{C}_{2}} \mathbf{u}_{2} \cdot \mathbf{d} \mathbf{r},
$$

where $\mathcal{C}_{i}$ denotes the part of the curve in the $i$ th fluid. Then, using the Euler equation in each fluid, it holds

$$
\begin{aligned}
\frac{d \Gamma}{d t} & =\int_{\mathcal{C}_{1}}\left\{\frac{d \mathbf{u}_{1}}{d t} \cdot d \mathbf{r}+\mathbf{u}_{1} \cdot d \mathbf{u}_{1}\right\}+\int_{\mathcal{C}_{2}}\left\{\frac{d \mathbf{u}_{2}}{d t} \cdot d \mathbf{r}+\mathbf{u}_{2} \cdot d \mathbf{u}_{2}\right\} \\
& =\left.\left(\frac{p}{\rho_{1}}+g z+\frac{\left|\mathbf{u}_{1}\right|^{2}}{2}\right)\right|_{Q_{1}} ^{Q_{2}}+\left.\left(\frac{p}{\rho_{2}}+g z+\frac{\left|\mathbf{u}_{2}\right|^{2}}{2}\right)\right|_{Q_{2}} ^{Q_{1}} \\
& =\left[p\left(Q_{2}\right)-p\left(Q_{1}\right)\right]\left(\frac{1}{\rho_{2}}-\frac{1}{\rho_{1}}\right) \\
& +\frac{1}{2}\left\{\left(\left|\mathbf{u}_{1}\left(Q_{2}\right)\right|^{2}-\left|\mathbf{u}_{2}\left(Q_{2}\right)\right|^{2}\right)-\left(\left|\mathbf{u}_{1}\left(Q_{1}\right)\right|^{2}-\left|\mathbf{u}_{2}\left(Q_{1}\right)\right|^{2}\right)\right\},
\end{aligned}
$$

where $p\left(Q_{i}\right)$ denotes the pressure at $Q_{i}$, and $\mathbf{u}_{j}\left(Q_{i}\right)$ denotes the velocity of the $j$ th fluid at the point $Q_{i}$, respectively, where $i, j=1,2$.

Let us first consider the traditional smooth interfacial waves of two layer flows with $\rho_{2}>\rho_{1}$. It is well-known [34 37] that, on the free surface of the interfacial waves, although the velocity normal to the interface is continuous, the tangential velocity is discontinuous, so that

$$
\left|\mathbf{u}_{1}\left(Q_{i}\right)\right| \neq\left|\mathbf{u}_{2}\left(Q_{i}\right)\right|, \quad i=1,2 .
$$

Besides, it holds $p\left(Q_{1}\right) \neq p\left(Q_{2}\right)$ in general. Thus, for the traditional interfacial waves with smooth crest, it holds $d \Gamma / d t \neq 0$ when the closed curve $\mathcal{C}$ crosses the interface. 
Therefore, the Kelvin theorem does not hold for the traditional interfacial waves with smooth crest.

However, for the peaked solitary waves governed by the UWM, we have $\rho_{1}=\rho_{2}$ so that the pressure term in (103) vanishes. Besides, due to the symmetry (13), it holds

$$
\left|\mathbf{u}_{1}\left(Q_{i}\right)\right|=\left|\mathbf{u}_{2}\left(Q_{i}\right)\right|, \quad i=1,2,
$$

so that the last term in (103) vanishes, too! Therefore, the Kelvin's theorem is still valid everywhere for the peaked solitary waves given by the UWM, although there exists the discontinuity at crest! This is indeed a little surprise: from the viewpoint of Kelvin's theorem, the peaked solitary waves are even more acceptable than the traditional interfacial waves of two-layer fluids with smooth crest!

\section{Concluding remarks and discussions}

Many wave models such as the Camassa-Holm $(\mathrm{CH})$ equation [6] admit peaked solitary waves, and thousands of articles about peaked solitary waves have been published. However, it was an open question whether the fully nonlinear wave equations admit peaked solitary waves or not. This is rather strange, since most of wave models such as the $\mathrm{CH}$ equation [6] were derived from the fully nonlinear wave equations under some assumptions.

In this paper, we propose a unified wave model (UWM) for progressive gravity waves with symmetric and permanent form in a finite water depth. The UWM is based on the symmetry and the fully nonlinear wave equations. Especially, unlike other wave models, its flow is unnecessary to be irrotational at crest $(x=0)$. Thus, the UWM is more general: it admits not only all traditional periodic and solitary waves with infinitely differential surface ( see $\S 3$ ), but also the peaked solitary waves (see $\S 4$ ) which include the famous peaked solitary wave (2) of the $\mathrm{CH}$ equation (1) and process many unusual characteristics. In addition, it is proved (see $\S 4.4$ ) that Kelvin's theorem still holds everywhere for the the peaked solitary waves, although there exists a vortex sheet at crest $(x=0)$. Therefore, the UWM unifies the traditional smooth waves and the peaked solitary ones, for the first time, to the best of the author's knowledge. In other words, the peaked solitary waves are consistent with the traditional smooth waves: they are as acceptable as the traditional smooth waves! It is fantastic that the two completely different types of waves can be derived from the same wave model, i.e. the UWM.

These peaked solitary waves expressed by the evanescent base-functions (26) have many unusual characteristics quite different from the traditional periodic and solitary waves expressed by the smooth base-functions (17). First, it has a peaked crest with a discontinuous vertical velocity at crest $(x=0)$. Secondly, it may be in the form of depression, corresponding to a negative wave height $H_{w}$, which has been reported for interfacial solitary waves but never for free-surface solitary waves, to the best of author's knowledge. Third, from free surface to bottom, its horizontal velocity always 
increases, and besides its kinetic energy either increases or decays a little. Especially, unlike the traditional smooth waves, which are dispersive with wave height that is a characteristic length of smooth wave in the vertical direction, the phase speed of peaked solitary waves has nothing to do with wave weight: in fact, they are dispersive (for a fixed wave height) with "the actual wavelength" $\lambda_{a}$, defined by (46), which is a characteristic length of peaked solitary wave in the horizontal direction. All of these are so different from the smooth periodic and solitary waves that they clearly indicate the novelty of the peaked solitary waves reported in this article.

All of these unusual characteristics come from the so-called evanescent basefunctions (26) of the peaked solitary waves, which are essentially different from the smooth base-functions (17) for the traditional periodic and solitary waves, although both of them automatically satisfy the Laplace equation (3) in $0<x<+\infty$, the bottom condition (6) and the bounded condition (12). It should be emphasized that both of them are widely used and can be found in the textbook of water waves [22], although the smooth base-functions (17) are currently more familiar and thus regarded as the mainstream. Note that the traditional base-functions (17) are infinitely differentiable everywhere, and automatically satisfy the symmetry conditions (13) and (14). However, the evanescent base-functions (26) have a discontinuous 1st-derivative (with respect to $x$ ) at crest $x=0$, and besides do not automatically satisfy the symmetry conditions (13) and (14). Mathematically, these essential differences of the two kinds of base-functions (17) and (26) are the origin of the completely different characteristics between the smooth and peaked waves.

Unlike traditional smooth waves, the peaked solitary waves admit not only the discontinuous 1st-derivative of elevation at crest but also the discontinuous velocity there: the vertical velocity $v$ changes sign as we cross the plane $x=0$. Thus, there exists a vorticity sheet at crest $x=0$. However, such kind of discontinuity of velocity is acceptable even in the frame of the traditional wave theories for smooth waves, since it also exists for the traditional interfacial waves expressed by the smooth base-functions (17): "the tangential velocity changes sign as we cross the surface" of interfacial waves, and "in reality the discontinuity, if it could ever be originated, would be immediately abolished by viscosity", as mentioned by Lamb [38] (§231, page 371). Similarly, in reality, the discontinuity of the vertical velocity (at $x=0$ ) of the peaked solitary waves, if it could ever be originated, would be immediately abolished by viscosity. So, in the frame of the inviscid fluid, the peaked solitary waves should be as reasonable as the widely accepted interfacial waves with smooth crest.

Besides, as shown in $\S 4.4$, for the traditional interfacial waves with smooth crest, a circulation (expressed by $\Gamma$ ) along a closed curve crossing the interface of two-layer fluids disobeys the Kelvin's theorem, i.e. $d \Gamma / d t \neq 0$. However, it is proved that the Kelvin's theorem still holds everywhere for the peaked solitary waves in general cases. Therefore, from the viewpoint of Kelvin's theorem, the peaked solitary waves are even more reasonable and thus more acceptable than the traditional interfacial waves with smooth crest.

In a sense, such kind of discontinuity (or singularity) of the peaked solitary waves 
can be removed. Let $U(z)$ denote the horizontal velocity of a progressive wave (with symmetry and permanent form) at crest $(x=0)$, governed by the UWM based on the symmetry and the fully nonlinear wave equations (3) - (12). Assume that, in the frame moving with the solitary wave, one could instantaneously replace the boundary $x=0$ by a porous vertical plate, and at the same time could enforce a horizontal velocity $U(z)=u(0, z)$ through the porous plate. Then, the corresponding velocity potential $\phi$ and wave elevation $\zeta(x)$ are governed by the same fully nonlinear wave equations (3) - (12), but only in the domain $x \in(0,+\infty)$. Therefore, for a properly given $U(z)$, one gains either the traditional periodic/solitary waves with smooth crest, if the smooth base-functions (17) are used, or the peaked solitary waves, if the evanescent base-functions (26) are employed, respectively*. In this case, the Laplace equation (3) is satisfied in the total physical domain $x \in(0,+\infty)$ so that no discontinuity (or singularity) exists at all, since $x=0$ becomes a physical boundary. For this wave propagation problem, the peaked solitary waves (although only in the region $0 \leq x<+\infty$ ) have very clear physical meanings and are physically as reasonable as the traditional smooth waves.

The peaked solitary surface waves found in this paper may provide us not only new explanations of some natural phenomenon but also a few theoretical predictions. First, the peaked solitary waves have an unusual and interesting characteristic: its phase speed has nothing to do with the wave height $H_{w}$. It should be emphasized that, according to the transcendental equation (28), the peaked solitary waves with a small wave height $H_{w}$ may propagate very quickly, since $\tan (k) / k \rightarrow+\infty$ as $k \rightarrow n \pi+\pi / 2$ for an integer $n \geq 0$. Thus, all of these peaked solitary waves with small wave height but different phase speed may create a huge solitary surface wave somewhere: this gives a new theoretical explanation about the so-called "rogue wave" that can suddenly appear on ocean, even when "the weather was good, with clear skies and glassy swells", as reported by Graham [54] and mentioned by Kharif [55]. Secondly, our peaked solitary waves predict free surface in the form of depression, corresponding to a negative wave height $H_{w}$. To the best of the author's knowledge, solitary waves of depression have been reported only for interfacial waves with smooth crest, but never for surface gravity waves. Such kind of peaked solitary surface waves of depression might be more difficult to create in practice and/or by experiment than the traditional ones with smooth crest. However, if this theoretical prediction would be physically correct, we should observe it in laboratory and/or in practice, sooner or later. This is an interesting but challenging work: it could enrich and deepen our understanding about solitary waves, no matter whether the conclusions are positive or not. Thirdly, according to the traditional wave theories, the kinetic energy of traditional waves decreases exponentially from free surface to bottom, thus a submarine far beneath surface is safe even if there are huge waves on ocean. However, different from traditional smooth periodic and solitary waves, the horizontal velocity of the peaked solitary waves always increases from free surface to bottom. Besides, its kinetic energy either increases or decays a little from free surface to bottom. However,

${ }^{*}$ Note that the corresponding horizontal velocities $U(z)$ at $x=0$ for the traditional smooth progressive waves with permanent form are different from that for the peaked solitary ones. 
due to the viscosity of fluid in practice, the horizontal velocity of water waves must be zero at bottom so that such kind of the peaked solitary waves might not exist exactly in its theoretical form as reported in this article, since there always exists a thin viscous boundary layer near bottom. However, if such kind of peaked solitary waves with large horizontal velocity and kinetic energy near bottom would indeed exist in practice, they should be quite dangerous to submarines, platforms and equipments in underwater engineering, even though they are not exactly in the same form as found in this paper.

Possibly, the new kind of peaked solitary waves might change some traditional view-points. For example, solitary waves are often regarded as a nonlinear phenomenon. However, we illustrate in $\S 4.1$ that solitary surface waves may exist even in a system of linear differential equations. Besides, it is widely believed that solitary water waves exist only in shallow water. But, we indicate here that solitary waves can exist even in a finite water depth, say, $D$ is unnecessary to be small. For instance, in the case of $D=100$ (meter), $k=1$ and the dimensionless wave height $H_{w}=0.05$, the corresponding peaked solitary wave propagates with the 5 meter wave-height in the phase speed $c=1.24796 \sqrt{g D} \approx 39.1$ meter per second, which is not very dangerous. However, in the case of $D=1000$ (meter) with the same dimensionless parameters (i.e. $k=1$ and $H_{w}=0.05$ ), the corresponding peaked solitary wave propagates with the 50 meter wave-height and the phase speed $c=123.5$ meter per second, which is destructive if it could indeed occur on the earth! Here, it may be worth mentioning that, the peaked solitary waves contain large kinetic energy only in a small domain near crest, since the kinetic energy either increases or decays a little from free surface to bottom, but decays exponentially in the horizontal direction. Thus, the peaked solitary waves should be more dangerous than the traditional smooth waves, if they could indeed exist in practice.

However, it should be emphasized that the peaked solitary waves found in this article are obtained under the assumptions that the fluid is inviscid and incompressible, the flow is irrotational in the domain $x>0$ and $x<0$ (it implies that the flow is not necessarily irrotational at $x=0$ ), the surface tension is neglected, and the wave elevation has a symmetry. Although part of these assumptions are used for the traditional periodic and solitary waves with smooth crest, the physical reasonableness of the peaked solitary waves should be studied deeply in future. Note that surface tension might have an important influence on the crest of the peaked solitary waves. Especially, the viscosity of fluid might have an important influence on the discontinuous vertical velocity and the vortex sheet at crest $(x=0)$ : it is unknown whether or not viscosity of fluid and surface tension might essentially change the properties of the peaked solitary waves. The author personally believes that these open questions can not be answered in the frame of inviscid fluid, and thus must be studied in the frame of viscous fluid. In the past, peaked solitary waves were investigated mainly from mathematical viewpoints. Now, it is the time to consider them more from physical viewpoints.

Note that the peaked solitary waves found in this article by the unified wave model logically include the famous peaked solitary wave (2) of the $\mathrm{CH}$ equation (1). 
It should be emphasized that the newly found peaked solitary waves have a discontinuous velocity and a vortex sheet at crest, and especially their phase speed has nothing to do with wave height. These unique characteristics are however not predicted by the traditional simplified wave models such as the $\mathrm{CH}$ equation. So, if the newly found peaked solitary waves are indeed physically correct and reasonable, they could deepen our understandings about the traditional peaked solitary waves. However, if these newly found peaked waves are physically not reasonable, the traditional peaked/cusped solitary waves given by these simplified wave models must be checked carefully from the physical viewpoints, although thousands of related articles have been published mainly from the mathematical viewpoints.

Note also that the symmetry plays an important role in the unified wave model (UWM). Such kind of symmetry has been successfully applied to give a theoretical explanation [56] for the newly found standing solitary waves by physical experiment [57]. Note that both of the odd and even symmetry were used in [56], but only even symmetry (13) is considered in this article. In fact, it is straightforward to include both of the odd and even symmetry in the UWM. However, it is a pity that such kind of symmetry does not exist for two or more progressive waves. This restricts the applications of the UWM based on the symmetry and fully nonlinear wave equations. To further extend the UWM, some new mathematical methods for discontinuous functions must be proposed.

Finally, this paper is a clear demonstration of the power of the HAM, for the discovery of the new kind of peaked solitary waves with many unusual characteristics, which have never been reported, to the best of the author's knowledge. Indeed, a truly new method always gives something new or different. So, this paper also illustrates the novelty of the HAM, too. Independent of small/large physical parameters, the HAM is a useful tool for lots of nonlinear problems.

Without doubts, further theoretical, numerical and experimental studies, and especially practical/experimental observations about the solitary surface waves with peaked crest, are needed in future. Indeed, discontinuity and/or singularity are more difficult to handle. But, they should not be evaded easily, since they might open some new fields of research, and greatly enrich and deepen our understandings about the real world.

\section{Acknowledgement}

Thanks to professor C.C. Mei (M.I.T., USA) for the helpful discussions, and professor Roger Grimshaw (Loughborough University, UK) for the discussion on the Kelvin's theorem for a closed curve crossing the interface of two layer fluids (about the traditional interfacial waves). This work is partly supported by the State Key Laboratory

of Ocean Engineering (Approval No. GKZD010061) and the National Natural Science Foundation of China (Approval No. 11272209). 


\section{References}

[1] J. S. Russell. On waves. Report of the fourteenth meeting of the British Association for the Advancement of Science, pages 311-390, 1845.

[2] J. Boussinesq. Théorie des ondes et des remous qui se propagent le long d'un canal rectangulaire horizontal, en communiquant au liquide contenu dans ce canal des vitesses sensiblement pareilles de la surface au fond. Journal de Math'ematiques Pures et Appliquées. Deuxiéme Série, 17:55 - 108, 1872.

[3] D. J. Korteweg and G. de Vries. On the change of form of long waves advancing in a rectangular canal, and on a new type of long stationary waves. Philosophical Magazine, 39:422 - 443, 1895.

[4] B. Benjamin, J.L. Bona, and J.J. Mahony. Model equations for long waves in nonlinear dispersive systems. Philos. Trans. Roy. Soc. London, 272:47 - 78, 1972.

[5] G.G. Stokes. On the theory of oscillation waves. Trans. Cambridge Phil. Phys., 8:441-455, 1894.

[6] R. Camassa and D. D. Holm. An integrable shallow water equation with peaked solitons. Phys. Rev. Lett., 71:1661 - 1664, 1993.

[7] A. Constantin. Existence of permanent and breaking waves for a shallow water equation: a geometric approach. Ann. Inst. Fourier, Grenoble, 50(2):321 - 362, 2000 .

[8] B. Fuchssteiner. Some tricks from the symmetry-toolbox for nonlinear equations: generalizations of the Camassa-Holm equation. Physica D, 95:296 - 343, 1996.

[9] Y.Y. Wu and K.F. Cheung. Explicit solution to the exact riemann problem and application in nonlinear shallow-water equations. Int. J. Numer. Meth. Fluids, $57: 1649-1668,2008$.

[10] R. Rosatti and L. Begnudelli. The riemann problem for the one-dimensional, free-surface shallow water equations with a bed step: Theoretical analysis and numerical simulations. J. Computational Phys., 229:760 - 787, 2010.

[11] S.J. Liao. Two kinds of peaked solitary waves of the KdV, BBM and Boussinesq equations. Science China- Physics, Mechanics \& Astronomy, 55:2469 - 2475, 2012.

[12] S.J. Liao. On peaked solitary waves of the Degasperis-Procesi equation. Science China- Physics, Mechanics \&3 Astronomy, 56:418 - 422, 2013.

[13] R. A. Kraenkel and A. Zenchuk. Camassa - Holm equation: transformation to deformed sinhGordon equations, cuspon and soliton solutions. J. Phys. A: Math. Gen., 32:4733 - 4747, 1999. 
[14] S.J. Liao. An approximate solution technique which does not depend upon small parameters (Part 2): an application in fluid mechanics. Int. J. Non-Linear Mechanics, 32:815-822, 1997.

[15] S.J. Liao. A uniformly valid analytic solution of $2 \mathrm{D}$ viscous flow past a semiinfinite flat plate. J. Fluid Mechanics, 385:101-128, 1999.

[16] S.J. Liao. Beyond Perturbation: Introduction to the Homotopy Analysis Method. CRC Press, Boca Raton, 2003.

[17] S.J. Liao. On the analytic solution of magnetohydrodynamic flows of nonnewtonian fluids over a stretching sheet. J. Fluid Mechanics, 488:189-212, 2003.

[18] S.J. Liao. Series solutions of unsteady boundary-layer flows over a stretching flat plate. Studies in Applied Mathematics, 117:239-263, 2006.

[19] S.J. Liao. Homotopy Analysis Method in Nonlinear Differential Equations. Springer \& Higher Education Press, Heidelberg, 2012.

[20] K. Vajravelu and R. Van Gorder. Nonlinear Flow Phenomena and Homotopy Analysis: Fluid Flow and Heat Transfer. Springer \& Higher Education Press, Heidelberg, 2013.

[21] E.D. Cokelet. Steep gravity waves in water of arbitrary uniform depth. Philosophical Transaction of the Royal Society of London - A, 286:286, 1977.

[22] C.C Mei, M. Stiassnie, and D.K.P. Yue. Theory and Applications of Ocean Surface Waves. World Scientific, New Jersey, 2005.

[23] J.W. Rayleigh. On waves. Phil. Mag., 1:257 - 271, 1876.

[24] J.D. Fenton. A ninth-order solution for the solitary wave. J. Fluid Mech., 53:257$271,1972$.

[25] J.D. Fenton. A high-order cnoidal wave theory. J. Fluid Mech., 94:129-161, 1979.

[26] W.D. McKee. Calculation of evenescent wave modes. Proc. ASCE, J. Waterway Port Coastal Ocean Eng., 114:373 - 378, 1988.

[27] S.R. Massel. Harmonic generation by waves propagating over a submerged step. Coastal Engineering, 7:357 - 380, 1983.

[28] J.T. Kirby and R.A. Dalrymple. Propagation of obliquely incident water waves over a trench. J. Fluid Mech., 133:47 - 63, 1983.

[29] J.T. Kirby. A general wave equation for waves over rippled beds. J. Fluid Mech., 162:171 - 186, 1986.

[30] D. Porter and D.J. Staziker. Extensions of the mild-slope equation. J. Fluid Mech., 300:367 - 382, 1995. 
[31] F. Mattioli. Resonant reflection of a series of submerged breakwaters. Nuovo Cimento, 13:823-833, 1990.

[32] F. Mattioli. Resonant refection of surface waves by non-sinusoidal bottom undulations. Appl. Ocean Res., 13:49 - 52, 1991.

[33] S.R. Massel. Extended refraction-diffration equation for surface waves. Coastal Engineering, 19:97 - 126, 1993.

[34] J. Grue, H.A. Friis, E. Palm, and P.O. Rusas. A method for computing unsteady fully nonlinear interfacial wave. J. Fluid Mech., 351:223 - 252, 1997.

[35] W. Choi and R. Camassa. Fully nonlinear internal waves in a two-fluid system. J. Fluid Mech., 396:1 - 36, 1999.

[36] T. Kataoka. Transverse instability of interfacial solitary waves. J. Fluid Mech., 611:255-282, 2008.

[37] M.R. Alam. A new triad resonance between co-propagating surface and interfacial waves. J. Fluid Mech., 691:267 - 278, 2012.

[38] H. Lamb. Hydrodynamics. Cambridge University Press, Cambridge, 1932.

[39] H. Blasius. Grenzschichten in flüssigkeiten mit kleiner reibung. Z. Math. u. Phys., $56: 1-37,1908$.

[40] M. Turkyilmazoglu. Purely analytic solutions of the compressible boundary layer flow due to a porous rotating disk with heat transfer. Physics of Fluids, 21:106104, 2009.

[41] H. Xu, Z.L. Lin, S.J. Liao, J.Z. Wu, and J. Majdalani. Homotopy-based solutions of the NavierStokes equations for a porous channel with orthogonally moving walls. Physics of Fluids, 22(5):053601, 2010.

[42] M. Turkyilmazoglu. An optimal analytic approximate solution for the limit cycle of Duffing - van der Pol equation. ASME J. Appl. Mech., 78:021005, 2011.

[43] Y.J. Li, B.T. Nohara, and S.J. Liao. Series solutions of coupled Van der Pol equation by means of homotopy analysis method. J. Mathematical Physics, 51:063517, 2010.

[44] S.J. Liao. A new branch of solutions of boundary-layer flows over an impermeable stretched plate. Int. J. Heat Mass Tran., 48:2529 - 2539, 2005.

[45] S.J. Liao and E. Magyari. Exponentially decaying boundary layers as limiting cases of families of algebraically decaying ones. Z. angew. Math. Phys., 57:777$792,2006$.

[46] S.P. Zhu. An exact and explicit solution for the valuation of american put options. Quant. Financ., 6:229 - 242, 2006. 
[47] S. J. Liao and K. F. Cheung. Homotopy analysis of nonlinear progressive waves in deep water. Journal of Engineering Mathematics, 45(2):105-116, 2003.

[48] L.W. Schwartz. Computer extension and analytic continuation of stokes' expansion for gravity waves. J. Fluid Mech., 62:553-578, 1974.

[49] M.S. Longuet-Higgins. Integral properties of periodic gravity waves of finite amplitudes. Proc. R. Soc. London - A, 342:157 - 174, 1975.

[50] L. B. Tao, H. Song, and S. Chakrabarti. Nonlinear progressive waves in water of finite depth - an analytic approximation. Coastal Engineering, 54(11):825-834, 2007.

[51] M.M. Rienecker and J.D. Fenton. A fourier approximation method for steady water waves. J. Fluid Mech., 104:119?37, 1981.

[52] B. Le Méhauté, D. Divoky, and A. Lin. Shallow water waves: a comparison of theories and experiments. Proceedings of 11th Conference on Coastal Engineering, pages $86-107,1968$.

[53] D.L. Xu, Z.L. Lin, S.J. Liao, and M. Stiassnie. On the steady-state fully resonant progressive waves in water of finite depth. J. Fluid Mechanics, 710:379 - 418, 2012.

[54] D.M. Graham. NOAA vessel swamped by rogue wave. Oceanspace, 2000. No. 284.

[55] C. Kharif and E. Pelinovsky. Physical mechanisms of the rogue wave phenomenon. Euro. J. Mech. B/Fluids, 22:603-634, 2003.

[56] S.J. Liao. Two new standing solitary waves in shallow water. Wave Motion, 50:785-792, 2013.

[57] J. Rajchenbach, A. Leroux, and D. Clamond. New standing solitary waves in water. Phys. Rev. Lett., 107:24502, 2011. 


\section{Appendix Detailed derivation of formulas (79) - (84)}

Write

$$
\left(\sum_{i=1}^{+\infty} \zeta_{i} q^{i}\right)^{m}=\sum_{n=m}^{+\infty} \mu_{m, n} q^{n}
$$

with the definition

$$
\mu_{1, n}(x)=\zeta_{n}(x), \quad n \geq 1
$$

Then,

$$
\left(\sum_{i=1}^{+\infty} \zeta_{i} q^{i}\right)^{m+1}=\left(\sum_{n=m}^{+\infty} \mu_{m, n} q^{n}\right)\left(\sum_{i=1}^{+\infty} \zeta_{i} q^{i}\right)=\sum_{n=m+1}^{+\infty} \mu_{m+1, n} q^{n}
$$

which gives

$$
\mu_{m, n}(x)=\sum_{i=m-1}^{n-1} \mu_{m-1, i}(x) \zeta_{n-i}(x), \quad m \geq 2, n \geq m .
$$

Define

$$
\psi_{i}^{n, m}(x)=\frac{\partial^{i}}{\partial x^{i}}\left(\left.\frac{1}{m !} \frac{\partial^{m} \phi_{n}}{\partial z^{m}}\right|_{z=0}\right) .
$$

By Taylor series, we have for any $z$ that

$$
\phi_{n}(x, z)=\sum_{m=0}^{+\infty}\left(\left.\frac{1}{m !} \frac{\partial^{m} \phi_{n}}{\partial z^{m}}\right|_{z=0}\right) z^{m}=\sum_{m=0}^{+\infty} \psi_{0}^{n, m} z^{m}
$$

and

$$
\frac{\partial^{i} \phi_{n}}{\partial x^{i}}=\sum_{m=0}^{+\infty} \frac{\partial^{i}}{\partial x^{i}}\left(\left.\frac{1}{m !} \frac{\partial^{m} \phi_{n}}{\partial z^{m}}\right|_{z=0}\right) z^{m}=\sum_{m=0}^{+\infty} \psi_{i}^{n, m} z^{m} .
$$

Then, on the unknown free surface $z=\eta(x ; q)$, we have using (106) that

$$
\begin{aligned}
\frac{\partial^{i} \phi_{n}}{\partial x^{i}} & =\sum_{m=0}^{+\infty} \psi_{i}^{n, m}\left(\sum_{s=1}^{+\infty} \zeta_{s} q^{s}\right)^{m}=\psi_{i}^{n, 0}+\sum_{m=1}^{+\infty} \psi_{i}^{n, m}\left(\sum_{s=m}^{+\infty} \mu_{m, s} q^{s}\right) \\
& =\sum_{m=0}^{+\infty} \beta_{i}^{n, m}(x) q^{m}
\end{aligned}
$$

where

$$
\begin{aligned}
\beta_{i}^{n, 0} & =\psi_{i}^{n, 0} \\
\beta_{i}^{n, m} & =\sum_{s=1}^{m} \psi_{i}^{n, s} \mu_{s, m}, \quad m \geq 1
\end{aligned}
$$


Similarly, on the unknown free surface $z=\eta(x ; q)$, it holds

$$
\begin{aligned}
\frac{\partial^{i}}{\partial x^{i}}\left(\frac{\partial \phi_{n}}{\partial z}\right) & =\sum_{m=0}^{+\infty} \gamma_{i}^{n, m}(x) q^{m}, \\
\frac{\partial^{i}}{\partial x^{i}}\left(\frac{\partial^{2} \phi_{n}}{\partial z^{2}}\right) & =\sum_{m=0}^{+\infty} \delta_{i}^{n, m}(x) q^{m}
\end{aligned}
$$

where

$$
\begin{aligned}
\gamma_{i}^{n, 0} & =\psi_{i}^{n, 1} \\
\gamma_{i}^{n, m} & =\sum_{s=1}^{m}(s+1) \psi_{i}^{n, s+1} \mu_{s, m}, \quad m \geq 1 \\
\delta_{i}^{n, 0} & =2 \psi_{i}^{n, 2} \\
\delta_{i}^{n, m} & =\sum_{s=1}^{m}(s+1)(s+2) \psi_{i}^{n, s+2} \mu_{s, m}, \quad m \geq 1 .
\end{aligned}
$$

Then, on the unknown free surface $z=\eta(x ; q)$, it holds using (112) that

$$
\begin{aligned}
\Phi(x, \zeta ; q) & =\sum_{n=0}^{+\infty} \phi_{n}(x, \zeta) q^{n}=\sum_{n=0}^{+\infty} q^{n}\left[\sum_{m=0}^{+\infty} \beta_{0}^{n, m}(x) q^{m}\right] \\
& =\sum_{n=0}^{+\infty} \sum_{m=0}^{+\infty} \beta_{0}^{n, m}(x) q^{m+n}=\sum_{s=0}^{+\infty} q^{s}\left[\sum_{m=0}^{s} \beta_{0}^{s-m, m}(x)\right] \\
& =\sum_{n=0}^{+\infty} \bar{\phi}_{n, 0}(x) q^{n},
\end{aligned}
$$

where

$$
\bar{\phi}_{n, 0}(x)=\sum_{m=0}^{n} \beta_{0}^{n-m, m} .
$$

Similarly, we have

$$
\begin{aligned}
\frac{\partial^{i} \Phi}{\partial x^{i}} & =\sum_{n=0}^{+\infty} \bar{\phi}_{n, i}(x) q^{n}, \\
\frac{\partial^{i}}{\partial x^{i}}\left(\frac{\partial \Phi}{\partial z}\right) & =\sum_{n=0}^{+\infty} \bar{\phi}_{n, i}^{z}(x) q^{n}, \\
\frac{\partial^{i}}{\partial x^{i}}\left(\frac{\partial^{2} \Phi}{\partial z^{2}}\right) & =\sum_{n=0}^{+\infty} \bar{\phi}_{n, i}^{z z}(x) q^{n},
\end{aligned}
$$


where

$$
\begin{aligned}
\bar{\phi}_{n, i}(x) & =\sum_{m=0}^{n} \beta_{i}^{n-m, m}, \\
\bar{\phi}_{n, i}^{z}(x) & =\sum_{m=0}^{n} \gamma_{i}^{n-m, m}, \\
\bar{\phi}_{n, i}^{z z}(x) & =\sum_{m=0}^{n} \delta_{i}^{n-m, m} .
\end{aligned}
$$

Then, on the unknown free surface $z=\eta(x ; q)$, it holds using (123) and (124) that

$$
\begin{aligned}
f & =\frac{1}{2} \nabla \Phi \cdot \nabla \Phi \\
& =\frac{1}{2}\left[\left(\frac{\partial \Phi}{\partial x}\right)^{2}+\left(\frac{\partial \Phi}{\partial z}\right)^{2}\right] \\
& =\sum_{m=0}^{+\infty} \Gamma_{m, 0}(x) q^{m},
\end{aligned}
$$

where

$$
\Gamma_{m, 0}(x)=\frac{1}{2} \sum_{n=0}^{m}\left(\bar{\phi}_{n, 1} \bar{\phi}_{m-n, 1}+\bar{\phi}_{n, 0}^{z} \bar{\phi}_{m-n, 0}^{z}\right) .
$$

Similarly, it holds on $z=\eta(x ; q)$ that

$$
\begin{aligned}
\frac{\partial f}{\partial x} & =\nabla \Phi \cdot \nabla\left(\frac{\partial \Phi}{\partial x}\right) \\
& =\frac{\partial \Phi}{\partial x} \frac{\partial^{2} \Phi}{\partial x^{2}}+\frac{\partial \Phi}{\partial z} \frac{\partial}{\partial x}\left(\frac{\partial \Phi}{\partial z}\right) \\
& =\sum_{m=0}^{+\infty} \Gamma_{m, 1}(x) q^{m}
\end{aligned}
$$

where

$$
\Gamma_{m, 1}(x)=\sum_{n=0}^{m}\left(\bar{\phi}_{n, 1} \bar{\phi}_{m-n, 2}+\bar{\phi}_{n, 0}^{z} \bar{\phi}_{m-n, 1}^{z}\right) .
$$

Besides, on $z=\eta(x ; q)$, we have by means of (123), (124) and (125) that

$$
\begin{aligned}
\frac{\partial f}{\partial z} & =\nabla \Phi \cdot \nabla\left(\frac{\partial \Phi}{\partial z}\right) \\
& =\frac{\partial \Phi}{\partial x} \frac{\partial}{\partial x}\left(\frac{\partial \Phi}{\partial z}\right)+\frac{\partial \Phi}{\partial z} \frac{\partial^{2} \Phi}{\partial z^{2}} \\
& =\sum_{m=0}^{+\infty} \Gamma_{m, 3}(x) q^{m}
\end{aligned}
$$


where

$$
\Gamma_{m, 3}(x)=\sum_{n=0}^{m}\left(\bar{\phi}_{n, 1} \bar{\phi}_{m-n, 1}^{z}+\bar{\phi}_{n, 0}^{z} \bar{\phi}_{m-n, 0}^{z z}\right) .
$$

Furthermore, using (123), (131) and (133), we have on $z=\eta(x ; q)$ that

$$
\nabla \Phi \cdot \nabla f=\frac{\partial \Phi}{\partial x} \frac{\partial f}{\partial x}+\frac{\partial \Phi}{\partial z} \frac{\partial f}{\partial z}=\sum_{m=0}^{+\infty} \Lambda_{m}(x) q^{m}
$$

where

$$
\Lambda_{m}(x)=\sum_{n=0}^{m}\left(\bar{\phi}_{n, 1} \Gamma_{m-n, 1}+\bar{\phi}_{n, 0}^{z} \Gamma_{m-n, 3}\right)
$$

Then, using (123), (124), (131) and (135), we have on $z=\eta(x ; q)$ that

$$
\begin{aligned}
& \mathcal{N}[\Phi(x, z ; q)] \\
= & \alpha^{2} \frac{\partial^{2} \Phi}{\partial x^{2}}+\frac{\partial \Phi}{\partial z}-2 \alpha \frac{\partial f}{\partial x}+\nabla \Phi \cdot \nabla f \\
= & \sum_{m=0}^{+\infty} \Delta_{m}^{\phi}(x) q^{m},
\end{aligned}
$$

where

$$
\Delta_{m}^{\phi}(x)=\alpha^{2} \bar{\phi}_{m, 2}+\bar{\phi}_{m, 0}^{z}-2 \alpha \Gamma_{m, 1}+\Lambda_{m}
$$

for $m \geq 0$.

Using (74) and (112), we have on $z=\eta(x ; q)$ that

$$
\begin{aligned}
\frac{\partial^{2}}{\partial x^{2}}\left(\Phi-\phi_{0}\right) & =\sum_{n=1}^{+\infty} \frac{\partial^{2} \phi_{n}(x, \eta)}{\partial x^{2}} q^{n}=\sum_{n=1}^{+\infty} q^{n}\left(\sum_{m=0}^{+\infty} \beta_{2}^{n, m} q^{m}\right) \\
& =\sum_{n=1}^{+\infty} q^{n}\left(\sum_{m=0}^{n-1} \beta_{2}^{n-m, m}\right),
\end{aligned}
$$

and similarly

$$
\begin{aligned}
\frac{\partial}{\partial z}\left(\Phi-\phi_{0}\right) & =\sum_{n=1}^{+\infty} \frac{\partial \phi_{n}(x, \eta)}{\partial z} q^{n}=\sum_{n=1}^{+\infty} q^{n}\left(\sum_{m=0}^{+\infty} \gamma_{0}^{n, m} q^{m}\right) \\
& =\sum_{n=1}^{+\infty} q^{n}\left(\sum_{m=0}^{n-1} \gamma_{0}^{n-m, m}\right),
\end{aligned}
$$

respectively. Then, on $z=\eta(x ; q)$, it holds due to the linear property of the operator (67) that

$$
\mathcal{L}\left(\Phi-\phi_{0}\right)=\sum_{n=1}^{+\infty} S_{n}(x) q^{n}
$$


where

$$
S_{n}(x)=\sum_{m=0}^{n-1}\left(\alpha^{2} \beta_{2}^{n-m, m}+\gamma_{0}^{n-m, m}\right)
$$

Then, on $z=\eta(x ; q)$, it holds

$$
(1-q) \mathcal{L}\left(\Phi-\phi_{0}\right)=(1-q) \sum_{n=1}^{+\infty} S_{n} q^{n}=\sum_{n=1}^{+\infty}\left(S_{n}-\chi_{n} S_{n-1}\right) q^{n}
$$

where

$$
\chi_{n}= \begin{cases}0, & \text { when } n \leq 1 \\ 1, & \text { when } n>1\end{cases}
$$

Substituting (143), (137) into (63) and equating the like-power of $q$, we have the boundary condition:

$$
S_{m}(x)-\chi_{m} S_{m-1}(x)=c_{\phi} \Delta_{m-1}^{\phi}(x), \quad m \geq 1 .
$$

Define

$$
\bar{S}_{n}(x)=\sum_{m=1}^{n-1}\left(\alpha^{2} \beta_{2}^{n-m, m}+\gamma_{0}^{n-m, m}\right)
$$

Then,

$$
S_{n}=\left(\alpha^{2} \beta_{2}^{n, 0}+\gamma_{0}^{n, 0}\right)+\bar{S}_{n}=\left.\left(\alpha^{2} \frac{\partial^{2} \phi_{n}}{\partial x^{2}}+\frac{\partial \phi_{n}}{\partial z}\right)\right|_{z=0}+\bar{S}_{n}
$$

Substituting the above expression into (145) gives the boundary condition on $z=0$ :

$$
\left.\left(\alpha^{2} \frac{\partial^{2} \phi_{m}}{\partial x^{2}}+\frac{\partial \phi_{m}}{\partial z}\right)\right|_{z=0}=\left.\left\{c_{\phi} \Delta_{m-1}^{\phi}+\chi_{m} S_{m-1}-\bar{S}_{m}\right\}\right|_{z=0}, \quad m \geq 1 .
$$

Substituting the series (5), (123) and (129) into (마), equating the like-power of $q$, we have on $z=0$ that

$$
\zeta_{m}(x)=\left.\left\{c_{\eta} \Delta_{m-1}^{\eta}+\chi_{m} \zeta_{m-1}\right\}\right|_{z=0}, \quad m \geq 1
$$

where

$$
\Delta_{m}^{\eta}=\zeta_{m}-\alpha \bar{\phi}_{m, 1}+\Gamma_{m, 0}
$$

